\title{
The interaction of thermocapillary convection and low-frequency vibration in nearly-inviscid liquid bridges
}

\author{
José A. Nicolás, Damián Rivas and José M. Vega
}

\begin{abstract}
The combined effect of thermocapillary stress and steady forcing due to vibrations of the disks in a model-half-zone axisymmetric liquid bridge is considered for low-viscosity liquids (i.e., with a large capillary Reynolds number), and low nondimensional vibration frequencies (i.e. small as comparex] to the capillary Reynolds number). An asymptotic model is derivexl for the slowly-varying streaming flow in the bulk (outside the oscillatory boundary layers) resulting from both effects that includes also buovancy and other thermal expansion effects. This model is used to first analyze the steady streaming flow patterus in isothermal conditions and then to show that mechanical vibrations can anuibilate almost completely thermocapillary flows of fairly large Reynolds numbers provided that: (i) the Prandtl number is appropriately small, (ii) both disks are vibrated, and (iii) the vibrating amplitudes, trequency and phases are appropriate (the counterbalancing effect depends crucially on the difference of the vibrating phases of both disks).
\end{abstract}

Mathematics Subject Classification (1991). Primary 76D05, 76D45; Secondary 76D30, $35 \mathrm{Q} 30,80 \mathrm{~A} 20$.

Keywords. Streaming flow, drift flow, thermocapillary convection, liquid bridges.

\section{Introduction and formulation}

In addition to their inherent interest as basic flow configurations, liquid bridges have been observed in flow through porous media [1-2] and particulates agglomeration [3], and are used for the experimental measurement of viscosity and surface tension [4]. They are also of crucial interest as simple models for the melt in unidirectional semiconductor crystal growth in microgravity conditions by means of the so-called float-zone technique [5-6]. In this case, thermocapillary effects are present in the melt and promote convective motions that are currently assumed to be responsible for the formation of undesirable non-uniformities in dopant distribution and crystal striations [7]. In order to improve crystal quality, several 
methods have been suggested to minimize thermocapillary convection, namely, float-zone rotation [8], application of magnetic fields [9-10] or gas jets [11], coating or partial covering of the melt [12] and, more recently, controlled vibration of the float-zone [13]. The latter is based on the well-known fact that mechanical vibrations produce steady (or slowly-varying) streaming flows. These flows (whose analysis goes back to Stokes [14]) have been receiving a continued attention in the literature (see, e.g., [15-18] and references given therein) but a systematic analysis of their interaction with thermocapillary effects is lacking; there are some relevant works concerned with the somewhat related problem associated with the effect of $g$-jitter on natural convection [19-20].

The main object of this paper is to derive an asymptotic model that includes the combined effect of vibration, thermocapillary stress and thermal expansion effects in the limiting case when the capillary Reynolds number $C^{-1}$ is large and the non-dimensional vibrating frequency $\Omega$ (based on the capillary time) is such that $\Omega \ll C^{-1}$. In addition, we shall use our asymptotic model to explain some experimental results and to predict some new ones. As we shall see both the derivation of the asymptotic model and its discussion include some subtle points that must be handled carefully. For the sake of clarity (and for its interest in applications) the liquid bridge configuration will be considered, but our analysis below extends straightforwardly to other configurations.

In order to formulate the problem we consider a liquid bridge of length $L$, held by surface tension between two horizontal circular, coaxial disks, that are held at fixed temperatures, $T_{0}$ and $T_{1}$, and are vibrating independently, with the same frequency, in the axial direction. The volume of the liquid equals that in the cylinder bounded by the disks and the free surface is assumed to be anchored at the edges of the disks. We assume that the viscosity $\mu$, the specific heat $c_{p}$ and the thermal conductivity $k$ are constant, but that density and surface tension depend linearly on temperature, as $\rho=\rho_{0}-\rho_{T}\left(T-T^{*}\right)$ and $\sigma=\sigma_{0}-\sigma_{T}\left(T-T^{*}\right)$, where $T^{*}=\left(T_{0}+T_{1}\right) / 2$ and the positive constants $\rho_{T}$ and $\sigma_{T}$ satisfy $\left|\rho_{T}\left(T_{1}-T_{0}\right)\right| \ll \rho_{0}$, $\left|\sigma_{T}\left(T_{1}-T_{0}\right)\right| \ll \sigma_{0}$. Now, density variations must be taken into account both in continuity and momentum conservation equations, and surface tension variations, in both the tangential and normal stress balances at the free surface; these effects will be taken into account only to the leading order. In addition, we assume that the density, viscosity and thermal conductivity of the surrounding gas are small as compared to the corresponding properties of the liquid; then the gas does not affect the dynamics of the liquid bridge and the free surface may be assumed to be thermally insulated. Since we are specifically interested in analyzing the interaction between thermocapillary convection and low-frequency vibration, in this paper we also neglect radiation losses (even though at very high temperaturess they could have some quantitative effect in the results, they are negligible in usual experimental conditions at low temperatures).

Under the assumptions above we use $R,\left(\rho_{0} R^{3} / \sigma_{0}\right)^{1 / 2}$ (i.e, the capillary time) and $\left(T_{1}-T_{0}\right) / 2$ to nondimensionalize length, time and the temperature difference 
$T-\left(T_{1}+T_{0}\right) / 2$. Then the governing equations (continuity and momentum and energy conservation) and boundary conditions (non-slipping, fixed temperature and anchorage of the free surface at the disks, smoothness of the velocity, pressure and temperature fields at the axis of symmetry, and kinematic compatibility, tangential and normal stress balances and no heat flux at the free surface) are

$$
\begin{aligned}
& u_{r}+r^{-1} u+w_{z}=\lambda\left(\theta_{t}+u \theta_{r}+w \theta_{z}\right), \\
& u_{t}+w\left(u_{z}-w_{r}\right)=-q_{r}-\left(q_{r}-u u_{r}-w w_{r}\right) \lambda \theta \\
& +C\left(u_{r r}+r^{-1} u_{r}-r^{-2} u+u_{z z}\right), \\
& w_{t}+u\left(w_{r}-u_{z}\right)=-q_{z}+B-\left(q_{z}-u u_{z}-w w_{z}\right) \lambda \theta \\
& +C\left(w_{r r}+r^{-1} w_{r}+w_{z s}\right) \text {, } \\
& \theta_{t}+u \theta_{r}+w \theta_{z}=(C / P)\left(\theta_{r r}+r^{-1} \theta_{r}+\theta_{z z}\right) \\
& u=0, \quad w=h_{ \pm}^{\prime}(t), \quad \theta=\mp 1, \quad f=1 \quad \text { at } z= \pm \Lambda+h_{ \pm}(t), \\
& u=w_{r}=q_{r}=\theta_{r}=0 \text { at } r=0 \text {, } \\
& u=f_{t}+f_{z} w \text { at } r=f \text {, } \\
& \left(w_{r}+u_{z}\right)\left(1-f_{z}^{2}\right)+2\left(u_{r}-w_{z}\right) f_{z}=-(C R e) \sqrt{1+f_{z}^{2}}\left(\theta_{z}+f_{z} \theta_{r}\right) \\
& \text { at } r=f \text {, } \\
& q-\frac{u^{2}+w^{2}}{2}+\left(1-\operatorname{ReC}^{2} \theta\right) \frac{f f_{z z}-1-f_{z}^{2}}{f\left(1+f_{z}^{2}\right)^{3 / 2}} \\
& =2 C \frac{u_{r}-\left(w_{r}+u_{z}\right) f_{z}+w_{z} f_{z}^{2}}{1+f_{z}^{2}} \text { at } r=f \text {, } \\
& \theta_{r}-f_{z} \theta_{z}=0 \text { at } r=f \text {, }
\end{aligned}
$$

with appropriate initial conditions (that are assumed to be axisymmetric). Here a cylindrical coordinate system $(v, \varphi, \vec{b})$ is used with associated unit vectors $e_{r}$, $e_{\varphi}, e_{z} ; u e_{r}+w e_{z}$ is the velocity, $q$ and $\theta$ are the stagnation pressure and the temperature, and the shape of the interface is given by $r=f(z, t)$. Notice that the total volume is conserved (as a consequence of (1.1), (1.6) and (1.7)) and, according to the assumption above equals $2 \pi \Lambda$, i.e.,

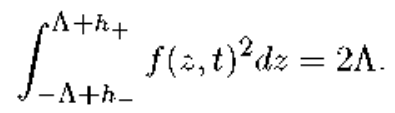

The problem (1.1)-(1.10) depends on (a) the slenderness of the bridge, $\Lambda=$ $L / 2 R$, that will be assumed to be of order unity, and smaller than $\pi$ to avoid the Rayleigh instability [21]; (b) the Bond number, $B= \pm \rho_{0} g R^{2} / \sigma_{0}( \pm g=$ axial gravitational acceleration) that will be assumed to be small; (c) the thermal expansion coefficient $\lambda=\rho_{T}\left|T_{1}-T_{0}\right| / 2 \rho_{0}$ that is usually quite small; (d) the capillary Reynolds number $C^{-1}=\left(\rho_{0} \sigma_{0} R\right)^{1 / 2} / \mu$, that uses to be quite large 
(the inverse is called the Ohnesorge or capillary number); (e) the thermocapillary Reynolds number $R \epsilon=\rho_{0} \sigma_{T}\left|T_{1}-T_{0}\right| R / 2 \mu^{2}$, that varies in practice from values of order unity to quite large values; (f) the Prondtl number $P=\mu c_{p} / k$, that also varies from fairly small values (for liquid metals) to large values (for most liquids); and $(\mathrm{g})$ the forcing functions $h_{+}$and $h_{-}$, that will be assumed to be small, and given by

$$
h_{ \pm}(t)=\sqrt{C} \beta_{ \pm} e^{i \Omega t}+c . c .,
$$

where $\beta_{+}$and $\beta_{-}$are complex and hereafter $c . c$. stands for the complex conjugate. Often the Marangon number $M a=R e \cdot P$ is used instead of the thermocapillary Reynolds.

For the sake of clarity the asymptotic model giving the steady streaming flow will be derived (in 32) in the distinguished limit

$$
C \ll 1, \quad \lambda=\alpha C, \quad B=b C, \quad\left|\beta_{ \pm}\right| \sim \Omega \sim R e \sim P \sim \alpha \sim|b| \sim 1,
$$

but this model will be valid for larger values of $\Omega$, namely, whenever

$$
1 \lesssim \Omega \ll C^{-1}
$$

and for larger values of $R e$, as it will be explained in $\$ 2.4$.

In a second distinguished limit (not considered in this paper) the vibrating frequency is large enough (namely, of the order of $C^{-1}$ ) as to excite a large amount of capillary eigenmodes that add up to a pair of capillary wavetrains that counterpropagate along the free surface. A linear analysis of those wavetrains (including their interaction with the disks) and of the associated steady streaming is given in $[22]$.

The paper is organized as follows. An asymptotic model will be obtained in $\$ 2$ that gives the time-averaged (in the capillary time scale) velocity in the bulk (i.e., outside the oscillatory boundary layers) in first approximation, and includes forcing terms due to vibration, thermocapillary stress and thermal expansion effects. The model and its validity limits will be discussed in $\S 2.4$, where several simplifications will be obtained that apply in some limiting cases of practical interest. As an application the asymptotic model will be numerically analyzed in $\S 3$ where we shall first consider the (fairly rich) structure of the drift flow in isothermal conditions and then look for effective control of thermocapillary convection. We shall elucidate that thermocinillary flows with a faimly large effectwe Reynolds number an be counterbalanced almost completely by vibrations provided that: (i) the Prandtl number is appropriately small, (ii) both disks are vibrated, (iii) the vibrating frequency is fairly large (as large as possible, but it cannot be too large, see (1.14)) and not too close to a natural frequency of the inviscid bridge, and (iv) the difference of the vibrating phases is appropriate (best results are obtained when that difference equals $\pi / 2$ ). Finally, some concluding remarks, concerning the applications, the limitations and some extensions of our results, will be drawn in $\S 4$. 


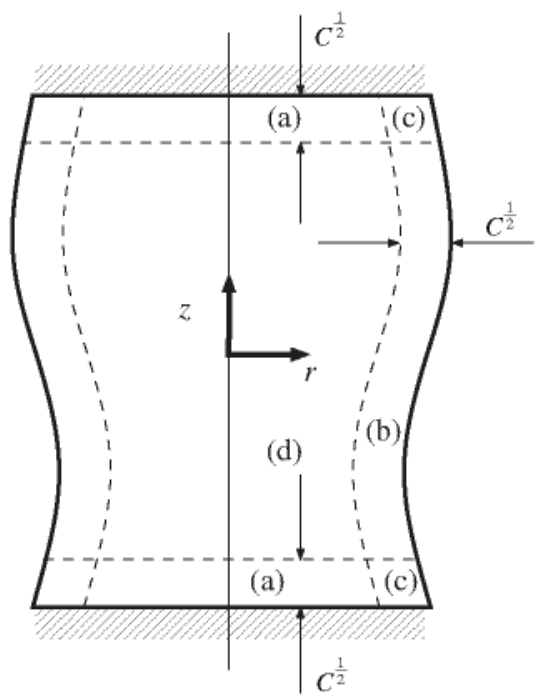

Figure 1.

A sketch of the four asymptotic regions in the liquid bridge.

\section{The asymptotic model}

In the limit (1.13) the mechanical and thermocapillary forcing terms in (1.5) and (1.8) are small (see (1.12) and (1.13)) and the pressure and velocity fields, and the shape of the free surface are close to their static values, $u=w=q-1-b C z=$ $0, f=1+O(C)$. Also, the capillary Reynolds number is large, and we must consider four distinguished regions in the liquid bridge (see (Fig. 1): (a) Two Stokes boundary layers near the disks; (b) an interface boundary layer near the free surface; (c) two corner regions near the border of the disks; and (d) the bulk, that is the remaining part of the liquid bridge. The characteristic size of regions (a), (b) and (c) (where inertia and viscous effects are comparable) is of the order of $C^{1 / 2}$. In fact, we shall not need to analyze the corner region (c) that would come into play only if higher order terms (than those considered below) were considered.

The main object of this section is to obtain an asymptotic model giving the streaming flow in the bulk. The streaming flow will be obtained upon averaging in the fast capillary time scale and thus will evolve (if it is nonsteady) in a slower time scale (see eq. (2.2) below). That flow will be driven by forcing terms resulting from vibration, thermocapillary stress and thermal expansion effects. In particular, vibrations produce (i) volumetric forcing terms in the bulk, which will be calculated in $\$ 2.1$, (ii) tangential forcing velocities at the edges of the Stokes boundary layers near the disks (first considered by Schlichting [23]), which will be calculated in $\$ 2.2$, and (iii) a tangential forcing stress at the edge of the interface boundary layer (first considered by Longuet-Higgins [24]), which will be 
calculated in 32.3 . The asymptotic model will be written in terms of the Eulerian mean velocity, resulting from the time-average of the velocity in the fast time scale associated with vibrations, and exhibits non-zero normal components at the edges of both the Stokes and the interface boundary layers when the vibrating flow is progressive. These normal components result from the fact that we shall work in a static reference frame and would disappear if working in a curvilinear, time-dependent reference frame attached to both the disks and the interface. This apparent paradox (for, the streaming flow should not change when using a second reference frame that is also static in the mean, in first approximation) is due to the fact that the Eulerian mean velocity is not the physically relevant one (as first pointed out by Stokes [14]); instead we must consider the Lagrangian mean velocity (also called drift [15] or mass-transport [24] velocity) that is, the velocity associated with the fast-time-average of the trajectories of material elements; the drift velocity is obviously the relevant one in connection with effective migration of material elements. The drift velocity does not coincide with the Eulerian mean velocity unless the vibrating flow is standing. Now, as we shall see in $\S 2.4$, the normal components of the drift velocity at the edges of the oscillatory boundary layers do always vanish and the apparent paradox disappears. At first sight, our arguments above should lead us to write the asymptotic model in terms of the drift velocity, but we will not do so because then the asymptotic model would be more involved from the mathematical point of view, as we shall explain in $\$ 2.4$.

\subsection{The bulk}

We shall seek for solutions of (1.1)-(1.12) such that the radial velocity and the shape of the free surface may be expanded in powers of $C^{1 / 2}$ as

$$
\begin{aligned}
u & =\sqrt{C} u_{1}^{0}(r, z, t, \tau)+\sum_{k=2}^{4} C^{k / 2}\left[u_{k}^{0}(r, z, t, \tau)+u_{k}^{s}(r, z, \tau)\right]+O\left(C^{5 / 2}\right), \\
f-1 & =\sqrt{C} f_{1}^{0}(z, t, \tau)+O(C) .
\end{aligned}
$$

The remaining variables $w, q-1-b C z$ and $\theta-\theta_{0}$ are expanded similarly to $u$, and the slow time variable $\tau$ is defined as

$$
\tau=C t .
$$

Also, for $k \geq 1$ the coefficient of $C^{k / 2}$ is decomposed in two parts; the first one will be called raprdly oscillatory because it oscillates in the fast time variable, and is required to give no contribution to the fast time average, i.e.,

$$
\int_{0}^{2 \pi / \Omega} u_{k}^{0} d t \equiv \int_{0}^{2 \pi / \Omega} w_{k}^{0} d t \equiv \int_{0}^{2 \pi / \Omega} q_{k}^{0} d t \equiv 0, \quad \int_{0}^{2 \pi / \Omega} f_{k}^{0} d t \equiv 0,
$$

while the second part will be called slowly varying because it does not depend on the fast time variable. Notice that we are assuming that the slowly varying parts of the pressure and velocity fields and of the shape of the interface vanish at order 
$C^{1 / 2}$; that assumption requires the initial vorticity to be of order $C$ in the bulk. Notice that $\left(C u_{2}^{s}, C w_{2}^{s}\right)$ gives a first approximation of the slowly varying part (or, equivalently, the Eulerian fast-time average) of the velocity field in the bulk.

Substitution of (2.1) into (1.1)-(1.4) yields, in $-\Lambda<\star<\Lambda, 0<r<1$,

$$
\begin{aligned}
u_{k r}^{0}+r^{-1} u_{k}^{0}+w_{k z}^{0}= & u_{k t}^{0}+q_{k r}^{0}=w_{k t}^{0}+q_{k z}^{0}=0 \\
u_{3 r}^{0}+r^{-1} u_{3}^{0}+w_{3 z}^{0}= & u_{2 r}^{s}+r^{-1} u_{2}^{s}+w_{2 z}^{s}=q_{2 r}^{s}=q_{2 z}^{s}=0 \\
u_{3 t}^{0}+q_{3 r}^{0}+w_{1}^{0}\left(u_{2 z}^{s}-\right. & \text { for } k=1 \text { and } 2 \\
& \left.+q_{2 z}^{s}\right)+\alpha q_{r}^{0} \theta_{0}=w_{3 t}^{0}+u_{1}^{0}\left(w_{2 r}^{s}-u_{2 z}^{s}\right)+\alpha q_{z}^{0} \theta_{0}=0 \\
u_{2 \tau}^{s}+w_{2}^{s}\left(u_{2 z}^{s}-w_{2 r}^{s}\right) & +\left[w_{1}^{0}\left(u_{3 z}^{0}-w_{3 r}^{0}\right)\right]^{s}=-q_{4 r}^{s}+\alpha\left(u_{1}^{0} u_{1 r}^{0}+w_{1}^{0} w_{1 r}^{0}\right)^{s} \theta_{0} \\
& -\alpha\left(q_{1 r}^{0} \theta_{1}^{0}\right)^{s}+u_{2 r r}^{s}+r^{-1} u_{2 r}^{s}-r^{-2} u_{2}^{s}+u_{2 z z}^{s} \\
w_{2 \tau}^{s}+u_{2}^{s}\left(w_{2 r}^{s}-u_{2 z}^{s}\right) & +\left[u_{1}^{0}\left(w_{3 r}^{0}-u_{3 z}^{0}\right)\right]^{s}=-q_{4 z}^{s}-b \alpha \theta_{0}+\alpha\left(u_{1}^{0} u_{1 z}^{0}+w_{1}^{0} w_{1 z}^{0}\right)^{s} \\
& -\alpha\left(q_{1 z}^{0} \theta_{1}^{0}\right)^{s}+w_{2 r r}^{s}+r^{-1} w_{2 r}^{s}+w_{2 z}^{s} \\
\theta_{0 \tau}+u_{2}^{s} \theta_{0 r}+w_{2}^{s} \theta_{0 z}+ & {\left[u_{1}^{0} \theta_{1 r}^{0}+w_{1}^{0} \theta_{1 z}^{0}\right]^{s}=P^{-1}\left(\theta_{0 r r}+r^{-1} \theta_{0 r}+\theta_{0 z z}\right) } \\
\theta_{1 t}^{0}+u_{1}^{0} \theta_{0 r}+w_{1}^{0} \theta_{0 z}= & 0
\end{aligned}
$$

where the super-script $s$ stands for the slowly varying part and it has been taken into account that (the rapidly oscillatory part of the vorticity at orders $C^{1 / 2}$ and $C$ identically vanishes in the bulk and), for $k=1$ and 2 ,

$$
u_{k r}^{0}-w_{k r}^{0} \equiv u_{k r r}^{0}+r^{-1} u_{k r}^{0}-r^{-2} u_{k}^{0}+u_{k z \tau}^{0} \equiv w_{k r r}^{0}+r^{-1} w_{k r}^{0}+w_{k z r}^{0} \equiv 0
$$

as it is readily obtained from (2.3)-(2.4). Notice that in Eqs. (2.7)-(2.9) appear steady terms resulting from the products of oscillatory terms.

Let us first consider the rapidly oscillatory parts of $u, w, q$ and $f$ at the leading order. If $\Omega$ is a natural frequency of the liquid bridge in the inviscid limit (i.e., if eqs. (2.12)-(2.16) below possess nontrivial solutions when $\beta_{+}=\beta_{-}=0$ ) then $q_{1}^{0}, u_{1}^{0}, w_{1}^{0}$ and $f_{1}^{0}$ are unbounded as $t \rightarrow \infty$ unless $\beta_{+}$and $\beta_{-}$are such that either $\beta_{+}+\beta_{-}=0$ or $\beta_{+}=\beta_{-}$(depending on whether $\Omega$ is associated with an even or an odd inviscid mode); that resonant case is considered in [25], where the effect of viscous dissipation and weak nonlinearity (that play an essential role near resonance) on the oscillatory field are also considered. Here we shall assume that $\Omega$ is not an inviscid natural frequency (in fact, we need to assume a little bit more than that, as it will be explained in \$2.4). If, in addition, the unforced inviscid natural modes (that decay exponentially in the time scale $\tau \sim 1$, see [25] or [26]) are ignored, then $u_{1}^{0}$, $w_{1}^{0}, q_{1}^{0}$ and $f_{1}^{0}$ may be written as

$$
\begin{gathered}
u_{1}^{0}=U(r, z) e^{i \Omega t}+c . c ., \quad w_{1}^{0}=W(r, \vec{s}) e^{i \Omega t}+c . c . \\
q_{1}^{0}=Q(r, z) e^{i \Omega t}+c . c ., \quad f_{1}^{0}=F(z) e^{i \Omega t}+c . c .
\end{gathered}
$$

where $U, W, Q$ and $F$ are uniquely determined by

$$
U_{r}+r^{-1} U+W_{z}=i \Omega U+Q_{r}=i \Omega W+Q_{z}=0,
$$




$$
\begin{gathered}
W=i \Omega \beta_{ \pm} \quad \text { at } z= \pm \Lambda, \\
U=W_{r}=0 \quad \text { at } r=0, \\
U-i \Omega F=Q+F+F^{\prime \prime}=0 \quad \text { at } r=1, \\
F( \pm \Lambda)=2 \int_{-\Lambda}^{\Lambda} F(z) d z+\beta_{+}-\beta_{-}=0 .
\end{gathered}
$$

Eqs. (2.12), (2.14) and (2.16) are obtained upon substitution of (2.1) and (2.11) into (1.1)-(1.3), (1.5)-(1.6) and (1.11), while in (2.13) and (2.15) we are anticipating matching conditions with the Stokes and the interface boundary layers.

The solution of (2.12)-(2.16) may be written as

$$
\begin{aligned}
U=(i / \Omega) Q_{r}, & W=(i / \Omega) Q_{2} \\
Q=\left(\beta_{+}+\beta_{-}\right) Q_{a}+\left(\beta_{+}-\beta_{-}\right) Q_{s}, & F=\left(\beta_{+}+\beta_{-}\right) F_{a}+\left(\beta_{+}-\beta_{-}\right) F_{s}
\end{aligned}
$$

where the antisymmetric (in the $z$ variable) functions $Q_{a}$ and $F_{a}$ and the symmetric functions $Q_{s}$ and $F_{s}$ are given by

$$
\begin{aligned}
& Q_{a}=\Omega^{2} \dot{s} / 2+\sum_{n \text { odd }} a_{n}\left[I_{0}\left(l_{n} r\right) / I_{0}\left(l_{n}\right)\right] \cos \left[l_{n}(z+\Lambda)\right], \\
& F_{a}=-\Omega^{2} z / 2+\delta_{a} \sin z+\sum_{n \text { odd }} a_{n}\left(l_{n}^{2}-1\right)^{-1} \cos \left[l_{n}(z+\Lambda)\right], \\
& Q_{s}=\Omega^{2}\left(2 z^{2}-r^{2}\right) / 8 \Lambda+b_{0}+\sum_{n \text { even }} b_{n}\left[I_{0}\left(l_{n} r\right) / I_{0}\left(l_{n}\right)\right] \cos \left[l_{n}(z+\Lambda)\right], \\
& F_{s}=\Omega^{2}\left(5-2 z^{2}\right) / 8 \Lambda+\delta_{s} \cos z-b_{0}+\sum_{n \text { even }} b_{n}\left(l_{n}^{2}-1\right)^{-1} \cos \left[l_{n}(z+\Lambda)\right],
\end{aligned}
$$

Here, the constants $\delta_{a}, \delta_{s}, a_{n}, b_{n}$ and $l_{n}$ are

$$
\begin{aligned}
\delta_{a} & =\Omega^{2} \frac{\Lambda^{2} / 2-\Omega^{2} \sum_{n \text { odd }} l_{n}^{-2}\left(\Omega^{2}+s_{n}\right)^{-1}}{\Lambda \sin \Lambda+2 \Omega^{2} \cos \Lambda \sum_{n \text { odd }}\left(\Omega^{2}+s_{n}\right)^{-1}\left(1-l_{n}^{2}\right)^{-1}}, \\
\delta_{s} & =\frac{1 / 4+\Omega^{2} \Lambda^{2} / 6-\Omega^{4} \sum_{n \text { even }} l_{n}^{-2}\left(\Omega^{2}+s_{n}\right)^{-1}}{\Lambda \cos \Lambda-\sin \Lambda-2 \Omega^{2} \sin \Lambda \sum_{n \text { even }}\left(\Omega^{2}+s_{n}\right)^{-1}\left(1-l_{n}^{2}\right)^{-1}}, \\
l_{n}=n \pi / 2 \Lambda, \quad s_{n}=l_{n}\left(1-l_{n}^{2}\right) I_{1}\left(l_{n}\right) / I_{0}\left(l_{n}\right) & \text { if } n \geq 1, \\
a_{n}=\left(\Omega^{2} / \Lambda\right)\left[2 \delta_{a} \cos \Lambda+\Omega^{2}\left(1-l_{n}^{2}\right) / l_{n}^{2}\right]\left(\Omega^{2}+s_{n}\right)^{-1} & \text { if } n \geq 1, \\
b_{0}=\left[\delta_{s} \sin \Lambda+\left(15 \Omega^{2}-2 \Omega^{2} \Lambda^{2}+6\right) / 24\right] / \Lambda, & \\
b_{n}=\left(\Omega^{2} / \Lambda\right)\left[2 \delta_{s} \sin \Lambda+\Omega^{2}\left(l_{n}^{2}-1\right) / l_{n}^{2}\right]\left(\Omega^{2}+s_{n}\right)^{-1} & \text { if } n \geq 2,
\end{aligned}
$$

and $I_{0}$ and $I_{1}$ are the two first modified Bessel functions.

Notice that $Q_{a}, Q_{s}, F_{a}$ and $F_{s}$ are real and thus the oscillation is standing (that is, the phase of the oscillating flow is independent of position) if $\beta_{+} \bar{\beta}_{-}=$real, or 
equivalently, if either (i) only one disk is vibrated $\left(\beta_{+} \cdot \bar{\beta}_{-}=0\right.$ ) or (ii) both disks are vibrated either in phase $\left(\beta_{+} \cdot \bar{\beta}_{-}=\right.$real and positive) or in counterphase $\left(\beta_{+} \cdot \bar{\beta}_{-}=\right.$real and negative). If instead both disks are vibrated with a phase shift different from 0 and $\pi$ (i.e., if imaginary part of $\beta_{+} \bar{\beta}_{-} \neq 0$ ) then the oscillation is progresstue.

The rapidly oscillatory parts of the velocity field at third order and of the temperature field at second order are readily obtained from $(2.6)$ and $(2.10)$ to be as given by

$$
u_{3}^{0}=U_{31} e^{i \Omega t}+c . c .+\mathbf{T H}, \quad w_{3}^{0}=W_{31} \epsilon^{i \Omega t}+c . c .+\mathbf{T H}, \quad \theta_{1}^{0}=\Theta \epsilon^{i \Omega t}+c . c .,
$$

where TH stands for the third harmonic (depending on the fast time variable as $\exp ( \pm 3 i \Omega t))$, and

$$
\begin{gathered}
U_{31}=i \Omega^{-1}\left[W\left(u_{2 z}^{s}-w_{2 r}^{s}\right)+Q_{31 r}+\alpha Q_{r} \theta_{0}\right], \\
W_{31}=i \Omega^{-1}\left[U\left(w_{2 r}^{s}-u_{2 z}^{s}\right)+Q_{31_{z}}+\alpha Q_{z} \theta_{0}\right], \\
\Theta=i \Omega^{-1}\left(U \theta_{0 r}+W \theta_{0 z}\right) .
\end{gathered}
$$

Notice that the velocity field $\left(U_{31}, W_{31}\right)$ has a non-zero vorticity. Substitution of $(2.11)-(2.12),(2.17)$ and $(2.23)-(2.25)$ into (2.7)-(2.9) yields

$$
\begin{aligned}
u_{2 r}^{s}+r^{-1} u_{2}^{s}+w_{2 z}^{s} & =0 \\
u_{2 \tau}^{s}+\left(w_{2}^{s}+w_{2}^{a}\right)\left(u_{2 z}^{s}-w_{2 r}^{s}\right) & =-q_{4 r}^{*}+\alpha\left(|U|^{2}+|W|^{2}\right) \theta_{0 r} \\
& +u_{2 r r}^{s}+r^{-1} u_{2 r}^{s}-r^{-2} u_{2}^{s}+u_{2 z}^{s} \\
u_{2 \tau}^{s}+\left(u_{2}^{s}+u_{2}^{a}\right)\left(w_{2 r}^{s}-u_{2 z}^{s}\right) & =-q_{4 z}^{*}-\alpha b \theta_{0}+\alpha\left(|U|^{2}+|W|^{2}\right) \theta_{0 z} \\
& +w_{2 r r}^{s}+r^{-1} w_{2 r}^{s}+w_{2 z z}^{s} \\
\theta_{0 \tau}^{s}+\left(u_{2}^{s}+u_{2}^{a}\right) \theta_{0 r}+\left(w_{2}^{s}+w_{2}^{a}\right) \theta_{0 z} & =P^{-1}\left(\theta_{0 r r}+r^{-1} \theta_{0 r}+\theta_{0 z z}\right)
\end{aligned}
$$

where we have also used the second equation (2.5) and

$$
\begin{gathered}
u_{2}^{a}=-i \Omega^{-1}(\bar{U} W)_{z}+c . c ., \quad w_{2}^{a}=i \Omega^{-1} r^{-1}(r \bar{U} W)_{r}+c c . \\
q_{4}^{*}=q_{4}^{s}+\left[i \Omega^{-1} U \bar{W}\left(u_{2 z}^{s}-w_{2 r}^{s}\right)+c c\right]+\alpha\left(|U|^{2}+|W|^{2}\right) \theta_{0} .
\end{gathered}
$$

These expressions for $u_{2}^{a}$, w $w_{2}^{a}$ and $q_{4}^{*}$ are obtained after some algebraic manipulations, when taking into account the first equation in (2.12) and that, according to the second and third equations in (2.12), $U_{z} \equiv W_{r}$. The asymptotic model giving $u_{2}^{s}, w_{2}^{s}, q_{4}^{*}$ and $\theta_{0}$ is posed by (2.26)-(2.29), with boundary conditions

$$
\begin{aligned}
& u_{2}^{s}=G^{ \pm}(r), \quad w_{2}^{s}=H^{ \pm}(r), \quad \theta_{0}=\mp 1 \quad \text { at } z= \pm \Lambda, \\
& u_{2}^{s}=w_{2 r}^{s}=q_{4 r}^{*}=\theta_{0 r}=0 \quad \text { at } r=0, \\
& u_{2}^{s}=\phi(z), \quad w_{2 r}^{s}=\psi(z)-\operatorname{Re} \theta_{0 z}, \quad \theta_{0 r}=0 \quad \text { at } r=1,
\end{aligned}
$$

and appropriate initial conditions, where (2.32) is obtained upon substitution of (2.1) into (1.6), while (2.31) and (2.33) (and the functions $G^{ \pm}, H^{ \pm}, \phi$ and $\phi$ ) 
will be obtained in $\$ \$ 2.2-2.3$ from matching conditions with the Stokes and the interface boundary layers. Notice that, as anticipated above, the velocity field $\left(u_{2}^{s}, w_{2}^{s}\right)$ exhibits normal components at the edges of both the Stokes boundary layers $\left(w_{2}^{s}=H^{ \pm}(r)\right)$ and the interface boundary layer $\left(u_{2}^{s}=\phi(s)\right)$, that will appear to be non-zero in general.

Finally, the components of the drift velocity at $r=r_{0}, z=z_{0}$, are calculated as [27] $u^{d}\left(r_{0}, \hat{\nu}_{0}, t, \tau\right)=\left[u(r(t), z(t), t, \tau]^{s}+O\left(C^{2}\right), \quad w^{d}\left(r_{0}, \hat{\sim}_{0}, t, \tau\right)=\right.$ $[w(r(t), z(t), t, \tau)]^{s}+O\left(C^{2}\right)$, where the super-script $s$ stands for the slowly varying part as above, and $r(t)=r_{0}+\int_{t_{0}}^{t} u(r(\sigma), z(\sigma), \sigma, \tau) d \sigma+O\left(C^{3 / 2}\right), \quad z(t)=$ $z_{0}+\int_{t_{0}}^{t} w(r(\sigma), z(\sigma), \sigma, \tau) d \sigma+O\left(C^{3 / 2}\right)$. If the expansions (2.1) and eqs. (2.11)(2.12) and (2.17) are replaced into those expressions then the following equations result

$$
\begin{array}{ll}
u^{d}=C u_{2}^{d}+O\left(C^{2}\right), & w^{d}=C w_{2}^{d}+O\left(C^{2}\right), \\
u_{2}^{d}=u_{2}^{s}+u_{2}^{a}, & w_{2}^{d}=w_{2}^{s}+w_{2}^{a},
\end{array}
$$

where $u_{2}^{a}$ and $w_{2}^{a}$ are as given by $(2.30)$. The correction $\left(u_{2}^{a}, w_{2}^{n}\right)$ is sometimes called Stokes drift. These two expressions and the asymptotic model (2.26)-(2.29), (2.31)-(2.33) will be discussed in $\$ 2.4$.

\subsection{The Stokes boundary layers}

For the sake of brevity we give details only for the boundary layer near $s=\Lambda$, where we use the stretched coordinate

$$
\xi=\left[z-\Lambda-\sqrt{C}\left(\beta_{+} e^{i \Omega t}+c . c\right)\right] / \sqrt{C}, \quad \text { with } \xi \sim 1
$$

and seek the expansions

$$
\begin{aligned}
u & =\sqrt{C}\left[\widetilde{U}_{1}(r, \xi) e^{i \Omega t}+c . c .\right]+C\left[\tilde{u}_{2}(r, \xi, \tau)+\tilde{u}_{2}^{0}(r, \xi, t, \tau)\right]+\cdots \\
w & =\sqrt{C}\left[i \Omega \beta_{+} e^{i \Omega t}+c . c .\right]+C\left[\widetilde{W}_{1}(r, \xi) e^{i \Omega t}+c c\right]+\cdots \\
q-1-b C z & =\sqrt{C}\left[\widetilde{Q}_{1}(r, \xi) e^{i \Omega t}+c . c .\right]+C\left[\tilde{q}_{2}^{s}(r, \xi, \tau)+\tilde{q}_{2}^{0}(r, \xi, t, \tau)\right]+\cdots \\
\theta & =\widetilde{\theta}_{0}(r, \xi, \tau)+\cdots
\end{aligned}
$$

as above, the rapidly oscillating parts of $u$ and $q$ at order $C$ are required to satisfy

$$
\int_{0}^{2 \pi / \Omega} \bar{u}_{2}^{0} d t \equiv \int_{0}^{2 \pi / \Omega} \tilde{q}_{2}^{0} d t \equiv 0
$$

while the slowly varying parts, $\tilde{u}_{2}^{s}$ and $\tilde{q}_{2}^{s}$, do not depend on $t$. Substitution of $(2.36)-(2.38)$ into $(1.1)-(1.5)$ leads to

$$
\tilde{\theta}_{0 \xi \xi}=\widetilde{Q}_{1 \xi}=\tilde{U}_{1 \xi \xi}-i \Omega \tilde{U}_{1}-\widetilde{Q}_{1 r}=\widetilde{W}_{1 \xi}+\tilde{U}_{1 r}+r^{-1} \tilde{U}_{1}=0,
$$




$$
\begin{aligned}
& \tilde{q}_{2 \xi}^{s}-\partial\left|\widetilde{U}_{1}\right|^{2} / \partial \xi=\tilde{u}_{2 \xi \xi}^{s}-\tilde{q}_{2 r}^{s}-\left(\widetilde{W}_{1} \widetilde{U}_{1 \xi}+c . c\right)=0, \\
& \tilde{\theta}_{0}+1=\widetilde{U}_{1}=\widetilde{W}_{1}=\tilde{u}_{2}^{s}=0 \quad \text { at } \xi=0 .
\end{aligned}
$$

Also, for the sake of brevity we anticipate the following matching requirements with the solution in the bulk

$$
\widetilde{\theta}_{0 \xi}=\widetilde{U}_{1 \xi}=\tilde{u}_{2 \xi}^{s}=0 \text { at } \xi=-\infty .
$$

When taking into account (2.42), integration of (2.39)-(2.41) yields

$$
\begin{gathered}
\widetilde{\theta}_{0}=-1, \quad \widetilde{U}_{1}=K(1-\Gamma) \\
\widetilde{W}_{1}=\left(K^{\prime}+r^{-1} K\right)\left[\frac{(1-i)(\Gamma-1)}{\sqrt{2 \Omega}}-\xi\right] \\
\tilde{u}_{2}^{s}=\bar{K} K^{\prime} \frac{|\Gamma|^{2}+1+2 i(\Gamma-\bar{\Gamma})}{2 \Omega} \\
+\bar{K}\left(K^{\prime \prime}+r^{-1} K\right) \frac{i\left(|\Gamma|^{2}-1\right)+2(1-2 i)(\bar{\Gamma}-1)+(1+i) \sqrt{2 \Omega} \xi \bar{\Gamma}}{2 \Omega}+c c
\end{gathered}
$$

where overbars and $c . c$. stand for the complex conjugate, $K=K(r)$ is an arbitrary function that is to be determined, and the function $\Gamma=\Gamma(\xi)$ is given by

$$
\Gamma(\xi)=\exp [(1+i) \sqrt{\Omega / 2} \xi] .
$$

Now, in order to apply matching conditions with the solution in the bulk, we take into account that the velocity components in the bulk satisfy

$$
\begin{gathered}
\theta\left(r, \Lambda+\sqrt{C}\left(\beta_{+} e^{i \Omega t}+c . c .\right)\right)=\theta_{0}(r, \Lambda)+O(\sqrt{C}) \\
u\left(r, \Lambda+\sqrt{C}\left(\beta_{+} e^{i \Omega t}+c . c .\right)\right)=\sqrt{C} U(r, \Lambda) e^{i \Omega t}+c . c . \\
+C\left[u_{2}^{0}(r, \Lambda, t, \tau)+u_{2}^{s}(r, \Lambda, \tau)\right]+O\left(C^{3 / 2}\right) \\
w\left(r, \Lambda+\sqrt{C}\left(\beta_{+} e^{i \Omega t}+c . c .\right)\right)=\sqrt{C} W(r, \Lambda) e^{i \Omega t}+c . c . \\
+C\left[w_{2}^{0}(r, \Lambda, t, \tau)+w_{2}^{s}(r, \Lambda, \tau)+\left(\beta_{+} e^{i \Omega t}+c . c .\right)\left(W_{z}(r, \Lambda) e^{i \Omega t}+c . c .\right)\right] \\
+O\left(C^{3 / 2}\right),
\end{gathered}
$$

(where we have used $(2.1),(2.11)$ and the identities $U_{z}(r, \Lambda) \equiv W_{r}(r, \Lambda) \equiv 0$ that are readily obtained from (2.12)-(2.13)), while the solution in this boundary layer is given by (2.37) (with $\widetilde{\theta}_{0}, \widetilde{U}_{1}, \vec{W}_{1}$ and $\tilde{u}_{2}^{s}$ as given in (2.43)-(2.44)), to obtain 


$$
\begin{aligned}
\theta_{0}(r, \Lambda) & =-1, \quad U(r, \Lambda)=K(r), \quad W(r, \Lambda)=i \Omega \beta_{+} \\
G^{+}(r) \equiv u_{2}^{s}(r, \Lambda, \tau) & =-\frac{3(1-i) \bar{U}(r, \Lambda) U_{r}(r, \Lambda)+c . c .}{2 \Omega}-\frac{2 r^{-1}|U(r, \Lambda)|^{2}}{\Omega} \\
H^{+}(r) \equiv w_{2}^{s}(r, \Lambda, \tau) & =-\left[\bar{\beta}_{+} W_{z}(r, \Lambda)+c . c\right] .
\end{aligned}
$$

Thus, the boundary conditions (2.13) and (2.31) at $z=\Lambda$ have been justified, and the functions $G^{+}$and $H^{+}$have been calculated. A similar analysis of the boundary layer near $z=-\Lambda$ yields

$$
\begin{gathered}
\theta_{0}(r,-\Lambda)=1, \quad W(r,-\Lambda)=i \Omega \beta_{-} \\
G^{-}(r)=-\frac{3(1-i) \bar{U}(r,-\Lambda) U_{r}(r,-\Lambda)+c . c .}{2 \Omega}-\frac{2 r^{-1}|U(r,-\Lambda)|^{2}}{\Omega} \\
H^{-}(r)=-\left[\bar{\beta}_{-} W_{z}(r,-\Lambda)+c . c\right] .
\end{gathered}
$$

\subsection{The interface boundary layer}

In this boundary layer we use the stretched coordinate

$$
\eta=[r-f(z, t, \tau)] / \sqrt{C},
$$

and seek the expansions

$$
\begin{aligned}
& u=f_{t}+C f_{\tau}+f_{z} w+C\left[\widetilde{U}_{1}(\eta, z) \epsilon^{i \Omega t}+c . c .\right] \\
& +C^{3 / 2}\left[\tilde{u}_{2}^{0}(\eta, z, t, \tau)+\tilde{u}_{2}^{s}(\eta, z, \tau)\right]+\cdots, \\
& w=\sqrt{C}\left(\widetilde{W}_{1} e^{i \Omega t}+\text { c.c. }\right)+C\left[\tilde{w}_{2}^{0}(\eta, \dot{s}, t, \tau)+\tilde{w}_{2}^{s}(\eta, z, \tau)\right] \\
& +C^{3 / 2}\left[\widetilde{w}_{3}^{0}(\eta, z, t, \tau)+\widetilde{w}_{3}^{s}(\eta, z, \tau)\right]+\cdots, \\
& q-1-b C z=\left(u^{2}+w^{2}\right) / 2+\sqrt{C}\left(\widetilde{P}_{1} e^{i \Omega t}+c . c .\right)+C\left[\tilde{p}_{2}^{0}(\eta, z, t, \tau)+\tilde{p}_{2}^{s}(\eta, z, \tau)\right] \\
& +C^{3 / 2}\left[\tilde{p}_{3}^{0}(\eta, z, t, \tau)+\tilde{p}_{3}^{s}(\eta, z, \tau)\right]+\cdots, \\
& \theta=\widetilde{\theta}_{0}(\eta, \hat{z}, \tau)+\sqrt{C}\left[\widetilde{\theta}_{1}^{0}(\eta, z, t, \tau)+\widetilde{\theta}_{1}^{s}(\eta, z, \tau)\right]+\cdots,
\end{aligned}
$$

where, as above, the rapidly oscillatory parts of $\theta, u, w$ and $q$ satisfy

$$
\begin{aligned}
\int_{0}^{2 \pi / \Omega} & { }_{1}^{0} d t=\int_{0}^{2 \pi / \Omega} u_{2}^{0} d t=\int_{0}^{2 \pi / \Omega} w_{2}^{0} d t \\
& =\int_{0}^{2 \pi / \Omega} q_{2}^{0} d t=\int_{0}^{2 \pi / \Omega} w_{3}^{0} d t=\int_{0}^{2 \pi / \Omega} q_{3}^{0} d t=0
\end{aligned}
$$


while the slowly varying parts, $\tilde{u}_{2}^{s}, \widetilde{w}_{2}^{s}, \widetilde{w}_{3}^{s}, \tilde{p}_{2}^{s}, \tilde{p}_{3}^{s}$ and $\widetilde{\theta}_{1}^{s}$ do not depend on $t$. Substitution of $(2.1),(2.11),(2.50)-(2.51)$ into $(1.1)-(1.4)$ and $(1.7)-(1.10)$ leads to the following equations

$$
\begin{aligned}
& \widetilde{\theta}_{0 \eta \eta}=\widetilde{\theta}_{1 \eta \eta}^{s}=\widetilde{P}_{1 \eta}=\widetilde{W}_{1 \eta \eta}-i \Omega \widetilde{W}_{1}-\widetilde{P}_{1 z}=\widetilde{U}_{1_{\eta}}+\widetilde{W}_{1 z}+i \Omega F=0, \\
& p_{2 \eta}^{0}-\Omega^{2}\left(F e^{i \Omega t}+c . c .\right)=\tilde{p}_{2 \eta}^{s}=\tilde{\omega}_{2 \eta \eta}^{s}-\tilde{p}_{2 z}^{s} \\
& -\left(\widetilde{\widetilde{U}}_{1} \widetilde{W}_{1 \eta}+\overline{\bar{W}}_{1} \widetilde{W}_{1 z}-\Omega^{2} \bar{F} F^{\prime}+c . c .\right)=0, \\
& \widetilde{w}_{2 \eta \eta}^{0}-\widetilde{w}_{2 t}^{0}-\tilde{p}_{2 z}^{0}=\left(\widetilde{U}_{1} \widetilde{W}_{1 \eta}+\widetilde{W}_{1} \widetilde{W}_{1 z}-\Omega^{2} F F^{\prime}\right) \epsilon^{2 i \Omega t}-\widetilde{W}_{1 \eta} e^{i \Omega t}+\text { c.c. } \\
& \hat{p}_{3 \eta}^{s}+\left[\bar{F}^{\prime}\left(i \Omega \vec{W}_{1}-\vec{W}_{1 \eta \eta}\right)+c . c\right]+\left(2 \overline{\vec{W}}_{1} i \Omega F^{\prime}+c . c .\right)=0 \\
& \widetilde{w}_{3 \eta \eta}^{s}-\tilde{p}_{3 z}^{s}=\bar{F}^{\prime}\left(2 \widetilde{W}_{1 \eta z}-\tilde{U}_{1 \eta \eta}+i \Omega \widetilde{U}_{1}\right)+\left(\bar{F}+\bar{F}^{\prime \prime}\right) \widetilde{W}_{1 \eta}+c . c . \\
& +(\Omega / 2 \pi) \int_{0}^{2 \pi / \Omega}\left[\left(\widetilde{W}_{1 \eta} e^{i \Omega t}+\text { c.c. }\right) \tilde{u}_{2}^{0}+\left(\widetilde{U}_{1} \epsilon^{i \Omega t}+\text { c.c. }\right) \widetilde{w}_{2 n}^{0}\right. \\
& \left.+\left(\widetilde{W}_{1 z} e^{i \Omega t}+c . c .\right) \tilde{w}_{2}^{0}+\left(\widetilde{W}_{1} e^{i \Omega t}+c . c .\right) \widetilde{w}_{2 z}^{0}\right] d t . \\
& +(\Omega / 2 \pi) \int_{0}^{2 \pi / \Omega}\left[\left(F^{\prime} e^{i \Omega t}+c . c .\right) f_{2 t t}^{0}-\Omega^{2}\left(F e^{i \Omega t}+c . c .\right) f_{2 z}^{0}\right] d t
\end{aligned}
$$

and the following boundary conditions at $\eta=0$

$$
\begin{aligned}
& \tilde{\theta}_{0 \eta}=\widetilde{\theta}_{1 \eta}^{s}=\widetilde{P}_{1}+F^{\prime \prime}+F=\widetilde{W}_{1 \eta}=\widetilde{U}_{1}=0 \\
& \tilde{p}_{2}^{0}+f_{2}^{0}+f_{2 z z}^{0}+\left[\left(F^{\prime 2} / 2-F^{2}\right) e^{2 i \Omega t}+c . c .\right] \\
& \quad=\tilde{p}_{2}^{s}+f_{2}^{s}+f_{2 z z}^{s}+\left|F^{\prime}\right|^{2}-2|F|^{2}=0 \\
& \widetilde{w}_{2 \eta}^{s}=\widetilde{w}_{2 \eta}^{0}+\left(i \Omega F^{\prime} e^{i \Omega t}+c . c\right)=0 \\
& \tilde{p}_{3}^{s}+f_{3}^{s}+f_{3 z z}^{s} \\
& \quad+(\Omega / 2 \pi) \int_{0}^{2 \pi / \Omega}\left[\left(F^{\prime} e^{i \Omega t}+c . c .\right) f_{2 z}^{0}-2\left(F \epsilon^{i \Omega t}+c . c .\right) f_{2}^{0}\right] d t=0 \\
& \widetilde{w}_{3 \eta}^{s}=\bar{F}^{\prime}\left(\widetilde{W}_{1 z}-\widetilde{U}_{1 \eta}\right)-\bar{F}^{\prime \prime} \widetilde{W}_{1}+c . c .-R e \widetilde{\theta}_{0 z}
\end{aligned}
$$

Also, for convenience we anticipate the following matching requirements with the solution in the bulk $\widetilde{\theta}_{0 \eta}=\widetilde{W}_{1 \eta}=\widetilde{w}_{2 \eta}^{s}=\widetilde{w}_{3 \eta \eta}^{s}=0$ at $\eta=-\infty$. When taking into account these requirements, integration of $(2.53)-(2.62)$ yields

$$
\begin{aligned}
& \tilde{\theta}_{0 \eta}=\tilde{\theta}_{1 \eta}^{s}=0, \quad \widetilde{P}_{1}=-\left(F+F^{\prime \prime}\right), \quad \widetilde{W}_{1}=-i\left(F^{\prime}+F^{\prime \prime \prime}\right) / \Omega, \\
& \tilde{U}_{1}=i\left(F^{\prime \prime}+F^{i v}-\Omega^{2} F\right) \eta / \Omega,
\end{aligned}
$$




$$
\begin{aligned}
& \tilde{p}_{2}^{0}=\mathrm{POL}, \quad \tilde{p}_{2}^{s}=\mathrm{POL}, \quad \widetilde{w}_{2 \eta}^{s}=\eta \mathrm{POL}, \\
& \widetilde{w}_{2}^{0}=-\sqrt{2 \Omega}\left[(1+i) F^{\prime} \Gamma(\eta) e^{i \Omega t}+\text { c.c. }\right]+\mathrm{POL}+\mathrm{ONRT}, \quad p_{3}^{s}=\mathrm{POL}, \\
& \widetilde{w}_{3 \eta}^{s}=i\left[3\left(2 \bar{F}^{i v}+2 \bar{F}^{\prime \prime}-\Omega^{2} \bar{F}\right) F^{\prime}+\left(\bar{F}^{\prime \prime}+\bar{F}^{\prime}\right) F^{\prime \prime}\right] / \Omega \\
& -2 i\left[\left(2 \bar{F}^{i v}+2 \bar{F}^{\prime \prime}-\Omega^{2} \bar{F}\right) F^{\prime}+\left(\bar{F}^{\prime \prime \prime}+\bar{F}^{\prime}\right) F^{\prime \prime}\right] \Gamma(\eta) / \Omega \\
& -(1-i) \sqrt{2 / \Omega}\left(\bar{F}^{i v}+\bar{F}^{\prime \prime}-\Omega^{2} \bar{F}\right) F^{\prime} \eta \Gamma(\eta)+c . c .-\operatorname{Re} \tilde{\theta}_{0 z}+\eta \mathrm{POL},
\end{aligned}
$$

where the function $\Gamma$ is as defined in (2.45), ONRT stands for oscillatory, nonresonant terms (depending on time as $\exp (i m \Omega t$.) with $m \neq 0, \pm 1$ ) and POL stands for a polynomial in the $\eta$ variable whose coefficients may depend on the remaining variables; in fact, when calculating the actual expressions for POL it may be seen that POL identically vanishes in the expressions giving $\tilde{w}_{2 \eta}^{s}$ and $\tilde{w}_{3 \eta}^{s}$.

Now we apply matching conditions between the solution in the bulk (2.1) and the solution in this boundary layer (2.51). To this end, we take into account that the solution in the bulk satisfies

$$
\begin{aligned}
\theta_{r}(f, z, t, \tau) & =\theta_{0 r}(1, z, \tau)+O(\sqrt{C}) \\
u(f, z, t, \tau) & =u(1, z, t, \tau)+(f-1) u_{r}(1, z, t, \tau)+O\left(C^{3 / 2}\right), \\
w_{r}(f, z, t, \tau) & =w_{r}(1, z, t, \tau)+(f-1) w_{r r}(1, z, t, \tau)+O\left(C^{3 / 2}\right), \\
q(f, z, t, \tau) & =q(1, z, t, \tau)+(f-1) q_{r}(1, z, t, \tau)+O\left(C^{3 / 2}\right),
\end{aligned}
$$

to obtain at $r=1$

$\theta_{0 r}=0, \quad U=i \Omega F, \quad Q=-\left(F+F^{\prime \prime}\right)$,

$\phi \equiv u_{2}^{s}=\bar{F}^{\prime} W-\bar{F} U_{r}+c . c$.

$\psi \equiv w_{2 r}^{s}=i\left[3\left(2 \bar{F}^{i \Downarrow}+2 \bar{F}^{\prime \prime}-\Omega^{2} \bar{F}\right) F^{\prime}+\left(\bar{F}^{\prime \prime}+\bar{F}^{\prime}\right) F^{\prime \prime}\right] / \Omega-\bar{F} W_{r r}+c . c(2.69)$

Thus, the boundary conditions (2.15) and (2.33) have been justified, and the functions $\phi$ and $\psi$ have been obtained.

\subsection{A discussion on the asymptotic model}

Let us now consider the asymptotic model (2.26)-(2.29), (2.31)-(2.33), with $G^{ \pm}$, $H^{ \pm}, \phi$ and $\psi$ as given by (2.46)-(2.49) and (2.68)-(2.69), and the expressions (2.35) for the drift velocity (with $u_{2}^{a}$ and $w_{2}^{a}$ as given by (2.30)).

a) In addition to the steady driving terms due to vibration near the disks and the interface appearing in the boundary conditions (2.31) and (2.33), eqs. (2.27)(2.29) exhibit volumetric forcing terms if $u_{2}^{a}$ and $w_{2}^{a}$ are non-zero. After looking at eqs. $(2.27)-(2.29)$ and $(2.35)$ we are tempted to rewrite eqs. (2.26)-(2.29) in terms 
of the drift velocity, intending to get rid of the volumetric driving terms. In fact, the vector field $\left(u_{2}^{a}, w_{2}^{a}\right)$ satisfies the continuity equation but it exhibits a non-zero vorticity and a non-zero Laplacian, and the additional terms that would be obtained in (2.27)-(2.28) do not derive from a potential; thus they cannot be eliminated by a re-definition of pressure. Then nothing is gained essentially, as it was already observed (implicitly) by Longuet-Higgins [24]. In addition, the new momentum equations would be somewhat misleading when considering the large-forcing-frequency limit, that will play a significant role below.

b) When $H^{ \pm}$and $\phi$ are non-zero the streamlines associated with $\left(u_{2}^{s}, w_{2}^{s}\right)$ intersect transversally both the (edges of the boundary layers near the) disks and the interface. But the flow associated with the drift velocity does not exhibit this curious phenomenon because $u_{2}^{d}(1, z)=\phi(z)+u_{2}^{a}(1, z) \equiv 0$ and $w_{2}^{d}(r, \pm \Lambda)=$ $H^{ \pm}(r)+\psi_{2}^{a}(r, \pm \Lambda) \equiv 0$, as readily seen when taking into account $(2.12)-(2.13)$, $(2.15),(2.30),(2.35),(2.47),(2.49)$ and $(2.68)$.

c) If $\beta_{+} \cdot \bar{\beta}_{-}$is real or, according to the remark right after eqs. $(2.17)-(2.22)$, if the oscillating flow is standing in first approximation, then

$$
\begin{aligned}
& u_{2}^{a} \equiv w_{2}^{a} \equiv 0, \quad H^{ \pm} \equiv 0 \quad \text { and } \phi \equiv \psi \equiv 0 \\
& \int_{0}^{1} G^{ \pm}(r) r d r=-\int_{0}^{1}|U(r, \pm \Lambda)|^{2} r d r / 2 \Omega<0,
\end{aligned}
$$

as seen upon substitution of (2.17)-(2.18) into (2.30), (2.46)-(2.49) and (2.68)(2.69), a further substitution of (2.46) and (2.48) into the left hand side of (2.71) and integration by parts (recall that $Q_{a}, Q_{s}, F_{a}$ and $F_{s}$ are real, that the phase of $U$ is constant and that $U(1, \pm \Lambda)=0$ ). Consequently, the streaming flow is driven only by the steady tangential velocity near the disks, whose associated net flux is negative (i.e., it drives the fluid towards the center of the disks). If instead the imaginary part of $\beta_{+} \cdot \bar{\beta}_{-}$is non-zero (i.e., if the oscillating flow is progressive) then none of the identities (2.70) holds and all forcing terms come into play; but some of the forcing terms dominate in some limiting cases that are considered below.

d) As the forcing frequency $\Omega$ approaches a natural frequency of the inviscid limit, $\tilde{\Omega} \sim 1,(U, W, Q, F)$ is seen to diverge as $|\Omega-\tilde{\Omega}|^{-1}$ times a fixed eigenmode associated with $\widetilde{\Omega}$ (that provides a standing oscillation), and $u_{2}^{a}, w_{2}^{a}$ and the forcing terms in (2.31)-(2.33) (defined by $(2.30),(2.46)-(2.49)$ and $(2.68)-(2.69))$ are seen to diverge as follows

$$
\left|G^{ \pm}\right| \sim|\Omega-\tilde{\Omega}|^{-2}, \quad\left|u_{2}^{a}\right| \sim\left|w_{2}^{a}\right| \sim\left|H^{ \pm}\right| \sim|\phi| \sim|\psi| \sim|\Omega-\check{\Omega}|^{-1} .
$$

In addition, when proceeding as in point (c) above, it is seen that

$$
G^{+} \simeq G^{-} \text {and } \int_{0}^{1} G^{ \pm}(r) r d r \simeq-\int_{0}^{1}|U(r, \pm \Lambda)|^{2} r d r / 2 \Omega<0 .
$$

Some care must be taken with this limit because $|\Omega-\bar{\Omega}|$ cannot be too small (esseritially, $|\Omega-\tilde{\Omega}| \gg C$ for $C \gtrsim 10^{-4}$, see [25] or [26]) for the oscillating flow to be inviscid in first approximation, as we are assuming in this paper. A fairly 
complete weakly-nonlinear analysis in the limit $|\Omega-\tilde{\Omega}| \sim C \rightarrow 0$ may be found in [25], where the associated streaming flow is also analyzed to conclude that it is not very effective to counterbalance thermocapillary convection.

e) As $\Omega \rightarrow \infty$ the inviscid oscillating flow defined by (2.11)-(2.16) exhibits a pair of counter-propagating capillary wavetrains, with a wavelength of the order of $\Omega^{-2 / 3}$, in a vicinity of the interface whose thickness is of the order of $\Omega^{-2 / 3}$, and an oscillating flow with a wavelength of order unity in the remaining part of the bulk. The associated functions $U, W$ and $Q$ are such that $|U| \sim$ $|W| \sim\left|\beta_{ \pm}\right| \Omega$ and $|Q| \sim\left|\beta_{ \pm}\right| \Omega^{2}$ except near the interface, where $|U| \sim|W| \sim$ $\left|\beta_{ \pm}\right| \Omega \log \Omega,|Q| \sim\left|\beta_{ \pm}\right| \Omega^{4 / 3} \log \Omega,|F| \sim\left|\beta_{ \pm}\right| \log \Omega$ and the spatial derivatives satisfy $\partial^{m+n} / \partial t^{m} \partial \iota^{n} \sim \Omega^{2(m+n) / 3}$ (see [22] for further details); in the sequel we shall ignore the factor $\log \Omega$ when dealing with orders of magnitude. Then, according to $(2.30),(2.46)-(2.49)$ and $(2.68)-(2.69)$, we have

$$
\left|u_{2}^{a}\right| \sim\left|w_{2}^{a}\right| \sim\left|\beta_{ \pm}\right|^{2} \Omega^{5 / 3}, \quad\left|H^{ \pm}\right| \sim\left|G^{ \pm}\right| \sim|\phi| \sim\left|\beta_{ \pm}\right|^{2} \Omega^{5 / 3}, \quad|\psi| \sim\left|\beta_{ \pm}\right|^{2} \Omega^{7 / 3},
$$

where the first four estimates apply only in a $O\left(\Omega^{-2 / 3}\right)$-neighborhood of the interface; in the remaining part of the bulk they must be replaced by the first four estimates below

$$
\left|u_{2}^{a}\right| \sim\left|w_{2}^{a}\right| \sim\left|\beta_{ \pm}\right|^{2} \Omega, \quad\left|H^{ \pm}\right| \sim\left|G^{ \pm}\right| \sim\left|\beta_{ \pm}\right|^{2} \Omega, \quad|U|^{2}+|W|^{2} \sim\left|\beta_{ \pm}\right|^{2} \Omega^{2} .
$$

Again (as in point (d)) some care must be taken with this limit because if $\Omega \gg 1$ and $0<C \ll 1$ then the above-mentioned capillary wavetrains are damped out by viscosity in a characteristic damping length that is of the order of $(\Omega C)^{-1}$ (see [22]), and viscous effects should not be ignored (as we are doing in this paper) unless the damping length is large as compared to the length of the liquid bridge (which is of order unity), that is, unless

$$
\Omega \ll C^{-1}
$$

f) The leading oscillatory terms in the expansions (2.1) must satisfy the linear equations and boundary conditions (2.12)-(2.16) for our analysis to be valid. If $\Omega \sim 1$ then this recuires the forcing amplitudes to be such that $\sqrt{C}\left|\beta_{ \pm}\right| \ll 1$, as is readily seen. If instead $\Omega \gg 1$ then a more subtle analysis (that is omitted here for the sake of brevity, see [22]) shows that nonlinear terms may be neglected provided that the following conditions are satisfied

$$
\Omega^{1 / 3} C\left|w_{2}^{s}(1, z)\right| \ll 1 \text { and } \sqrt{C}\left|\beta_{ \pm}\right| \Omega \ll 1
$$

g) If the tangential stress at the free surface, given by the second boundary condition (2.33) is large then the streaming flow exhibits a viscous boundary layer, whose thickness (that is of the order of (tangential stress) ${ }^{-1 / 3}$ ) must be large as compared to the thickness of the oscillatory boundary layer (that is of the order of $(C / \Omega)^{1 / 2}$ ) for our asymptotic model to be valid. That condition imposes two restrictions that are seen to be less stringent than (2.77) (after a cautious analysis that is omitted for the sake of brevity). 
h) Let us now consider the combined limit

$$
\begin{gathered}
1 \ll \Omega \ll C^{-1}, \quad R e^{2 / 3} P=O(1), \quad \Omega^{1 / 3} C R e^{2 / 3} \ll 1 \\
\left|\beta_{ \pm}\right|^{2} \ll \Omega^{5 / 3}, \quad\left|\beta_{ \pm}\right|^{2} \Omega^{7 / 3} \sim R e \\
|\alpha b| \ll R e^{4 / 3}, \quad \alpha\left|\beta_{ \pm}\right|^{2} \Omega^{2} \ll R e^{4 / 3}
\end{gathered}
$$

which will be exploited below, in $\$ 3.3$, to obtain conditions when vibration does counterbalance the thermocapillary flow. In the pure thermocapillary flow (i.e., in the absence of vibrations) we have $\left|u_{2}^{s}\right| \sim\left|w_{2}^{s}\right| \sim 1$ if $R e \sim 1$. If, instead, $R e \gg 1$ then the thermocapillary flow exhibits a viscous boundary layer near the interface, whose thickness is of the order of $R e^{-1 / 3}$, where $\left|u_{2}^{s}\right| \sim R e^{1 / 3}$ and $\left|w_{2}^{s}\right| \sim R e^{2 / 3}$; outside this boundary layer, we have

$$
\left|u_{2}^{s}\right| \sim\left|w_{2}^{s}\right| \sim R e^{2 / 3} .
$$

Notice that these estimates also hold if $R e \sim 1$. Now, all estimates below concerning volumetric forcing terms will be made outside the boundary layer that will have only a localized effect. Similarly, since $\Omega$ is large, there is a small, $\Omega^{-2 / 3}$-neighborhood of the interface (where the estimates (2.74) hold) that will be also ignored; consequently, the estimates (2.75) will be used. If, in addition the estimates (2.78)-(2.80) are taken into account, then we readily obtain that

$$
\begin{aligned}
& \left|u_{a}\right| \ll\left|u_{2}^{s}\right|, \quad\left|w_{a}\right| \ll\left|w_{2}^{s}\right|, \\
& \left|\alpha\left(|U|^{2}+|W|^{2}\right) \theta_{0 r}\right| \ll\left|w_{2}^{s}\left(u_{2 z}^{s}-w_{2 r}^{s}\right)\right|, \\
& \left|-\alpha b \theta_{0}+\alpha\left(|U|^{2}+|W|^{2}\right) \theta_{0 z}\right| \ll\left|u_{2}^{s}\left(w_{2 r}^{s}-u_{2 z}^{s}\right)\right|,
\end{aligned}
$$

and therefore, (i) the volumetric forcing terms associated with $u_{2}^{a}$ and $w_{2}^{a}$ may be ignored in (2.27)-(2.29), and (ii) the volumetric forcing terms associated with thermal expansion may also be ignored in (2.27)-(2.28). In addition, the forcing velocities at the edge of the oscillatory boundary layers near the disks $\left(G^{ \pm}\right.$and $H^{ \pm}$, see (2.31)) and the forcing normal velocity at the edge of the oscillary boundary layer near the interface ( $\phi$, see (2.33)) may be also neglected, according to (2.74)(2.75) and (2.78)-(2.80). Finally, notice that the validity limits (2.77) are satisfied, according to $(2.78)-(2.80)$.

To summarize our results above, the asymptotic model applies in the asymptotic limit (2.78)-(2.79) (i.e., the validity limits are satisfied) and it may be simplified by taking

$$
u_{2}^{a}=w_{2}^{a}=0, \quad \alpha=0, \quad G^{ \pm}=H^{ \pm}=0, \quad \phi=0
$$

in first approximation. 


\section{Steady flow in the bulk}

\subsection{The streaming flow in the bulk}

For convenience, the forcing complex amplitudes are written in terms of the associated real amplitudes and phases as

$$
\beta_{+}=a_{+} \exp \left(i \nu_{+}\right), \quad \beta_{-}=a_{-} \exp \left(i \nu_{-}\right),
$$

and subscripts are dropped out to rewrite the asymptotic model posed by (2.26)$(2.29),(2.31)-(2.33)$, and the expressions $(2.30),(2.35),(2.48)-(2.49)$ and $(2.68)-$ (2.69) for the drift velocity $\left(u^{d}, w^{d}\right)$, the Stokes drift $\left(u^{a}, w^{a}\right)$ and the forcing functions $G^{ \pm}, H^{ \pm}, \phi$ and $\psi$ as follows

$$
u^{d}=u^{s}+u^{a}, \quad w^{d}=w^{s}+w^{a} .
$$

If sub-scripts are dropped out then the resulting model for the slowly-varying streaming (i.e., the fast-time averaged) flow in the bulk is written as

$$
\begin{aligned}
& u_{r}^{s}+r^{-1} u^{s}+w_{z}^{s}=0, \\
& u_{\tau}^{s}+u^{s} u_{r}^{s}+w^{s} u_{z}^{s}+w^{a}\left(u_{z}^{s}-w_{r}^{s}\right)=-p_{r}+\alpha K \theta_{r}+u_{r r}^{s}+r^{-1} u_{r}^{s}-r^{-2} u^{s}+u_{z z}^{s} \\
& w_{\tau}^{s}+u^{s} w_{r}^{s}+w^{s} w y_{z}^{s}+u^{a}\left(w_{r}^{s}-u_{z}^{s}\right)=-p_{z}-\alpha b \theta+\alpha K \theta_{z}+w_{r r}^{s}+r^{-1} w_{r}^{s}+w_{z z}^{s}, \\
& \theta_{\tau}+\left(u^{s}+u^{a}\right) \theta_{r}+\left(w^{s}+w^{a}\right) \theta_{z}=P^{-1}\left(\theta_{r r}+r^{-1} \theta_{r}+\theta_{z z}\right), \\
& u^{s}=G^{ \pm}(r), \quad w^{s}=H^{ \pm}(r), \quad \theta=\mp 1 \quad \text { at } s= \pm \Lambda \\
& u^{s}=w_{r}^{s}=p_{r}=\theta_{r}=0 \quad \text { at } r=0, \\
& u^{s}=\phi(s), \quad w_{r}^{s}=-\operatorname{Re} \theta_{z}+\psi(\Delta), \quad \theta_{r}=0 \quad \text { at } r=1,
\end{aligned}
$$

where we now use the pressure $p=q_{4}^{*}-\left[\left(u_{2}^{s}\right)^{2}+\left(w_{2}^{s}\right)^{2}\right] / 2$, instead of the stagnation pressure $q_{4}^{*}$, and $K(r, z)=|U(r, \vec{z})|^{2}+|W(r, z)|^{2}$. The Stokes drift components $u^{a}$ and $w^{i}$ and the forcing functions $K, G^{ \pm}, H^{ \pm}, \phi$ and $\psi$ are

$$
\begin{aligned}
u^{a}= & 4 \Omega^{-3}\left(Q_{a r} Q_{s z}-Q_{a z} Q_{s r}\right)_{z} a_{+} a_{-} \sin \left(\nu_{+}-\nu_{-}\right), \\
w^{a}= & -4 \Omega^{-3} r^{-1}\left(r Q_{a r} Q_{s z}-r Q_{a z} Q_{s r}\right)_{r} a_{+} a_{-} \sin \left(\nu_{+}-\nu_{-}\right), \\
K= & \Omega^{-2}\left[\left(Q_{a r}+Q_{s r}\right)^{2}+\left(Q_{a z}+Q_{s z}\right)^{2}\right] a_{+}^{2} \\
& +\Omega^{-2}\left[\left(Q_{a r}-Q_{s r}\right)^{2}+\left(Q_{a z}-Q_{s z}\right)^{2}\right] a_{-}^{2} \\
& +2 \Omega^{-2}\left(Q_{a r}^{2}-Q_{s r}^{2}+Q_{a z}^{2}-Q_{s z}^{2}\right) a_{+} a_{-} \cos \left(\nu_{+}-\nu_{-}\right), \\
G^{ \pm}= & -\Omega^{-3}\left(g_{1} \pm g_{2}\right)\left[3 g_{1}^{\prime} \pm 3 g_{2}^{\prime}+2 r^{-1}\left(g_{1} \pm g_{2}\right)\right] a_{+}^{2} \\
& -\Omega^{-3}\left(g_{1} \mp g_{2}\right)\left[3 g_{1}^{\prime} \mp 3 g_{2}^{\prime}+2 r^{-1}\left(g_{1} \mp g_{2}\right)\right] a_{-}^{2} \\
& +2 \Omega^{-3}\left[3 g_{1} g_{1}^{\prime}-3 g_{2} g_{2}^{\prime}+2 r^{-1}\left(g_{1}^{2}-g_{2}^{2}\right)\right] a_{+} a_{-} \cos \left(\nu_{+}-\nu_{-}\right) \\
& \pm 6 \Omega^{-3}\left(g_{1} g_{2}^{\prime}-g_{1}^{\prime} g_{2}\right) a_{+} a_{-} \sin \left(\nu_{+}-\nu_{-}\right), \\
H^{ \pm}= & 2 \Omega^{-1}\left[ \pm g_{2}^{\prime}-g_{1}^{\prime}+r^{-1}\left( \pm g_{2}-g_{1}\right)\right] a_{+} a_{-} \sin \left(\nu_{+}-\nu_{-}\right),
\end{aligned}
$$




$$
\begin{aligned}
\phi= & 4 \Omega^{-1}\left[F_{a}^{\prime} F_{s}^{\prime \prime \prime}-F_{s}^{\prime} F_{a}^{\prime \prime \prime}+F_{a}\left(F_{s}^{i v}+F_{s}^{\prime \prime}\right)\right. \\
& \left.-F_{s}\left(F_{a}^{i v}+F_{a}^{\prime \prime}\right)\right] a_{+} a_{-} \sin \left(\nu_{+}-\nu_{-}\right), \\
\psi= & 4 \Omega^{-1} g_{3} a_{+} a_{-} \sin \left(\nu_{+}-\nu_{-}\right),
\end{aligned}
$$

where the functions $g_{1}, g_{2}$ and $g_{3}$ are

$$
\begin{aligned}
& g_{1}(r) \equiv Q_{s r}(r, \Lambda), \quad g_{2}(v)=Q_{a r}(v, \Lambda) \\
& g_{3}(z) \equiv\left[F_{s}^{\prime \prime}(z)+F_{s}^{\prime \prime \prime}(z)\right] F_{a}(z)-\left[F_{a}^{\prime \prime}(z)+F_{a}^{\prime \prime \prime}(z)\right] F_{s}(z) \\
& \quad+2\left[3 F_{s}^{i v}(s)+3 F_{s}^{\prime \prime}(s)-\Omega^{2} F_{s}(s)\right] F_{a}^{\prime}(z)-2\left[3 F_{a}^{i v}(z)+3 F_{a}^{\prime \prime}(z)-\Omega^{2} F_{a}(s)\right] F_{s}^{\prime}(z) \\
& \quad+\left[F_{s}^{\prime \prime \prime}(z)+F_{s}^{\prime}(z)\right] F_{a}^{\prime \prime}(z)-\left[F_{a}^{\prime \prime \prime}(z)+F_{a}^{\prime}(s)\right] F_{s}^{\prime \prime}(z),
\end{aligned}
$$

with the functions $Q_{s}, Q_{a}, F_{s}$ and $F_{a}$ as given by (2.19)-(2.22).

Notice that the model depends on the slenderness $\Lambda$, on the vibrating frequency $\Omega$, amplitudes, $a_{+}$and $a_{-}$, and phase shift $\nu_{+}-\nu_{-}$, the thermocapillary Reynolds number Re, the Prandtl number $P$ and the re-scaled Bond number $b$ and thermal expansion coefficient $\alpha$, which according to (1.13) are given by

$$
\alpha=\lambda C^{-1}, \quad b=B C^{-1}
$$

There are too many parameters involved and completely general conclusions concerning their influence on the associated streaming flow patterns are beyond the scope of this paper. Instead, the steady flow patterns associated with the asymptotic model (3.3)-(3.9) will be numerically obtained below only in some limiting cases of practical interest when, according to our estimates in $\$ 2.4$, several further simplifications apply. We shall compare our results with experimental results in the literature, in both isothermal and non-isothermal conditions, and predict some new behaviors.

\subsection{Numerical method}

The numerical method used in this paper for the computation of the steady flow presents the following main features. The pressure-velocity formulation is considered and the discretization is made using the finite volume method of Patankar [28]. The Pressure Correction Method, see Connell and Stow [29], is used to solve the discretized equations obtained from the governing equations. In particular, the SIMPLEC approximation of Van Doormal and Raithby [30] is used. Concerning the coupling of the temperature and flow fields, a decoupled method of solution is used, where at each iteration step the temperature field is updated by solving the energy equation for the current flow field.

A central difference scheme is used in this work in the discretization of the governing equations [31]. In performing the computations, the domain is divided into small control volumes, whose size follows a logarithmic distribution that concertrates the control volumes near the disks and the free surface (i.e., in the viscous boundary layers when the Reynolds number associated with the flow is large). The 


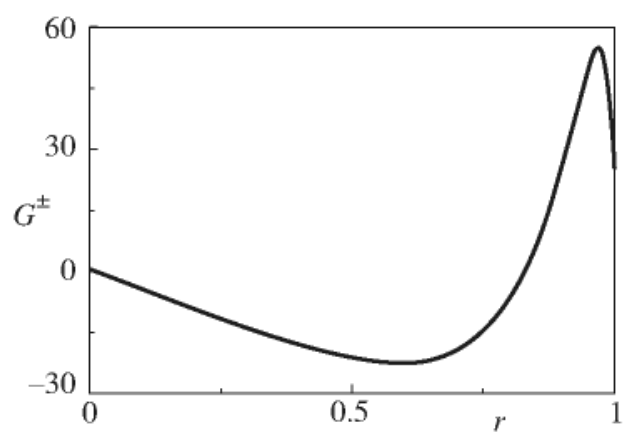

Figure 2.

The forcing steady radial velocities near the disks, $G^{+}$and $G^{-}$, for $\Lambda=1, \Omega=3.2656$, $a_{+}=$ $a_{-}=10^{-5 / 2}$ and $\nu_{+}-\nu_{-}=-\pi / 2$.

centers of these control volumes form the grid used for scalar variables, and the staggered grid system of Harlow and Welch [32] is considered for the components of the velocity vector.

In the iterative procedure convergence is declared when the four variables, $\theta$, $u, v$ and $p$, verify simultaneously the following relative error criterion

$$
\frac{\sum\left|g_{i j}-g_{i j}^{0}\right|}{\sum\left|g_{i j}\right|}<\varepsilon,
$$

where the summation is over all control volumes, and $g_{i j}^{0}$ represents the value of the appropriate variable $g_{i j}$ at the previous step. In this work the value $\varepsilon=5 \cdot 10^{-5}$ is considered. Also, following the nomenclature of Van Doormaal and Raithby [30], the value $E=8$ is used for the relaxation parameter. The number of control volumes chosen for the discretization of the domain $[-\Lambda, \Lambda] \times[0,1]$ is $200 \times 100$.

\subsection{Streaming flows in isothermal conditions $(R e=\alpha=0)$}

In the absence of temperature gradients the energy equation (3.6) is decoupled and may be ignored, and the volumetric driving terms resulting from thermal expansion (i.e., the coefficients of $\alpha$ in (3.4)-(3.5)) may be also ignored. For the sake of brevity we shall only consider in detail two limiting cases in which the vibrating flow is standing in first approximation. These two cases have been selected because they are the only ones that have been experimentally analyzed in the literature, in [33].

a) As the forcing fequency $\Omega$ approaches a natural frequency of the inviscid limit $\tilde{\Omega}$, the forcing terms and the Stokes drift satisfy the estimates (2.72) (recall that the divergence is due to the fact that we neglected viscous dissipation in the analysis of the oscillating flow, and that our analysis breaks down if $|\Omega-\tilde{\Omega}| \sim C$ ). Thus, we may take

$$
u^{a}=w^{a}=0, \quad H^{ \pm}=0, \quad \phi=\psi=0
$$


(a)

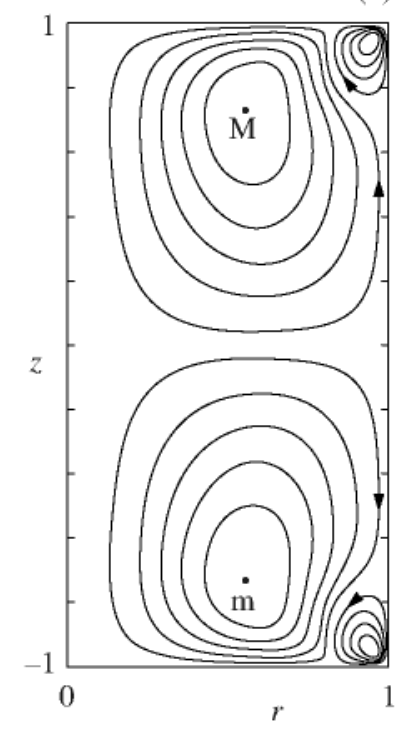

(b)

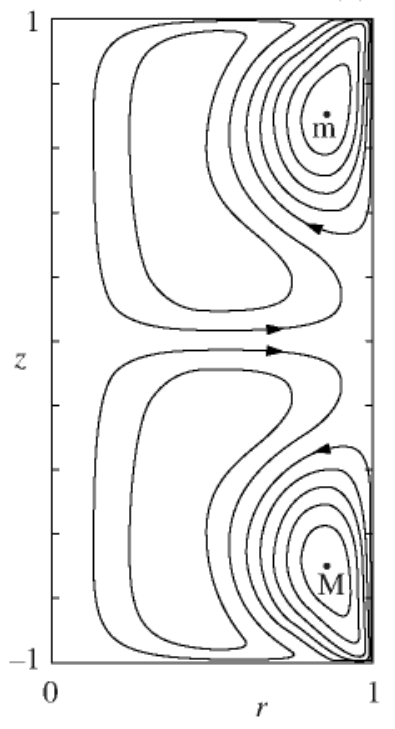

Figure 3 .

Streamlines in the bulk for $\Lambda=1, \Omega=3.2656, \nu_{-}-\nu_{+}=\pi / 2$ and (a) $a_{+}=a_{-}=10^{-5 / 2}$ and (b) $a_{+}=a_{-}=10^{-2}$. Represented are 10 equispaced streamlines, see Table 1 for extreme values.

in first approximation and the only forcing term is that appearing in the first boundary condition (3.5), with $G^{ \pm}$as given in (3.13). In addition the drift velocity coincides with $\left(u^{s}, w^{s}\right)$, according to (3.2) and (3.19), that is

$$
u^{d}=u^{s} \quad \text { and } \quad w^{d}=w^{s}
$$

in first approximation. Since $C$ may be as small as $10^{-4}$ for liquid metals, we may take $\Omega-\tilde{\Omega}=10^{-3}$. For illustration, the forcing functions $G^{+}$and $G^{-}$are plotted in Fig. 2 for $\Lambda=1, a_{+}=a_{-}=10^{-5 / 2}, \nu_{-}-\nu_{+}=\pi / 2$ and $\Omega=3.2656 \simeq \tilde{\Omega}+10^{-3}$, where $\tilde{\Omega}$ is the first inviscid eigenfrequency. Notice that $G^{+}$and $G^{-}$are indistinguishable, according to (2.73). The streamlines of the resulting streaming flow are given in Fig. 3a. Notice that (i) the flow is essentially symmetric and that (ii) it exhibits two small (toroidal) eddies near the edge of the disks that push the liquid away from the disks along the interface, and two larger (toroidal) counterrotating eddies (that push the liquid towards the disks along the interface). This was to be expected; the small eddies are due to the fact that the radial forcing velocity at the disks is positive in a small region near the corners (see Fig. 2), and the larger eddies are due to the fact that the net forcing radial flux is negative (see (2.73)). This flow pattern seems to be in qualitative agreement with the experimental observations in [33, Fig. 12]. In particular, the flow is symmetric and the main eddies push the liquid towards the disks along the interface, as in [33]; the small eddies near the corners, instead, are not observed in the pictures in [33]. Notice that this 
rough agreement is independent of the fact that only one disk was vibrated in the experiment in [33] while the results in Fig. 3 are for two sided vibration, because at resonance the response of the bridge consists of the associated eigenmode (that is qualitatively independent of the way it is excited) in first approximation. For larger forcing amplitudes the size of the small eddies increases due to convective terms, as shown in Fig. 3b; this second flow pattern was not observed by Mollot et al. [33]. Those two (essentially symmetric) flow patterns are obviously not the best ones to counterbalance the (qualitatively antisymmetric) thermocapillary flow generated by temperature gradients resulting from fixing the temperature of the disks, as we are doing in this paper. But there is a more essential feature that makes these flows inconvenient also to counterbalance symmetric thermocapillary flows, namely, the price for any possible counterbalancing effect is to introduce large tangential velocities near the disks. Our conclusions above stand for other values of $\Lambda$ and $\tilde{\Omega}$, and they do match with the results of the analysis of the drift flow resulting from weakly-nonlinear, almost-resonant oscillations [25].

b) If either (i) both disks are vibrated either in phase $\left(\nu_{+}=\nu_{-}\right)$or in counterphase $\left(\nu_{+}=\nu_{-} \pm \pi\right)$ or (ii) only one disk is vibrated (i.e., if $a_{+} a_{-}=0$ ), then $\left(\beta_{+} \cdot \bar{\beta}_{-}=\right.$real and, according to $\left.(2.70)\right)$ the equations $(3.19)-(3.20)$ are identically satisfied. Thus the flow is driven only by the tangential forcing velocities at the edge of the Stokes boundary layers (appearing in the first boundary condition (3.7) and given in (3.13)). For illustration, the forcing tangential velocities $G^{+}$ and $G^{-}$(see (3.7) and (3.13)) are plotted in Fig. 4 for $\Lambda=1, \Omega=\left(\Omega_{n}+\Omega_{m+1}\right) / 2$ $(\simeq 5.394,10.1604,15.7876,22.1225,29.1105,36.6909$ for $m=1, \ldots, 6)$, where $\Omega_{n}$ is the eigenfrequency associated with the $m$-th inviscid mode, in three cases: (i) $a_{+}=a_{-}=1, \nu_{+}-\nu_{-}=0$, (ii) $a_{+}=a_{-}=1, \nu_{-}-\nu_{+}=\pi$ and (iii) $a_{+}=1$, $a_{-}=0$; for other values of the slenderness, the plots of $G^{+}$and $G^{-}$have been seen to be qualitatively similar to those in $\mathrm{Fig}$. 4 . The streamlines associated with the streaming flow, resulting from the forcing tangential velocities given in Fig. 4a for $m=2$, are given in Fig. 5a. Notice that this symmetric flow exhibits three pairs of eddies resulting from the three bumps in the graph of $G^{+}=G^{-}$(see Fig. 4a); for larger values of the forcing amplitudes the upper eddies near the edge of the disks enlarge (due to convective effects) to dominate the other two pairs of eddies. If the forcing terms, $G^{+}=G^{-}$, are as those in Fig. 4a for $m=6$, then the resulting streamlines are essentially as those in Figs. $3 \mathrm{a}$ and $3 \mathrm{~b}$ (for small and larger forcing amplitudes respectively), the only difference being that now the flow exhibits also two new pairs of counter-rotating eddies, of an extremely small size, near the edge of the disks; these new eddies are due to the last two bumps (near $r=1$ ) in the graph of $G^{+}=G^{-}$, that enclose a very small area. The plots in Fig. 4a for $m$ odd are completely similar to that in Fig. 2; thus the resulting flows exhibit streamlines that are as those in Figs. 3a or $3 \mathrm{~b}$ (depending on the forcing amplitude). The forcing terms for $a_{+}=a_{-}$and $\nu_{-}-\nu_{+}=\pi$ (see Fig. 4b) are similar to those in Fig. 4a and thus produce flow patterns that are similar to those described above. Concerning the case $a_{+} \neq 0, a_{-}=0$, the associated flow pat- 
terns are non-symmetric and may be described as some kind of mixture of those described above. For illustration, the streamlines of the flow associated with the case $a_{+}=1, a_{-}=0, m=1$ (see Fig. 4c) are plotted in Fig. 5b; notice that this flow pattern is clearly non-symmetric and that it exhibits a remarkable feature, namely, the flow is stronger near $(z=-\Lambda)$ the disk that is not vibrated (as shown by the small separation between the equispaced streamlines); this effect has been also numerically detected for other values of $\Lambda$ and $\Omega$. As the forcing frequency $\Omega$ moves from $\left(\Omega_{m}+\Omega_{m+1}\right) / 2$ to either $\Omega_{m}$ or $\Omega_{m+1}$ the flow pattern becomes more and more symmetric (for as $\left|\Omega-\Omega_{m}\right| \ll 1$ the streamlines are essentially symmetric, as explained in point (a) above). For illustration, the forcing velocities $G^{+}$and $G^{-}$and the streamlines for $\Omega=4$ (that is in between of $\Omega_{1} \simeq 3.2656$ and $\left.\left(\Omega_{1}+\Omega_{2}\right) / 2 \simeq 5.394\right)$ are plotted in Fig. 6 . Notice that this flow pattern is more symmetric, as that in [33, Fig. 14].

Again, as in case (a) (and for the same reasons), these flows are not appropriate to counterbalance thermocapillary convection.

c) If $a_{+} a_{-} \neq 0,\left|\nu_{+}-\nu_{-}\right|$is neither close to 0 nor close to $\pi$, then the approximation (3.18) does not apply and the forcing terms associated with $u^{a}, w^{a}, H^{ \pm}$ and $\phi$ must be taken into account. When the forcing frequency $\Omega$, the forcing amplitudes $a_{+}$and $a_{-}$and the phase shift $\nu_{+}-\nu_{-}$are varied we obtain a large variety of qualitatively different flow patterns (that include those considered in points (a) and (b) above as limiting cases) whose analysis is beyond the scope of this paper. Here we only point out that, as in cases (a) and (b), these flows are not expected to be the appropriate ones to annihilate thermocapillary convection because again the price for any counterbalancing effect is to introduce tangential drift velocities at the edges of the Stokes boundary layers (equal to $G^{ \pm}(r)+u^{a}(r, \pm \Lambda$ ), see (3.2) and (3.7)) that are not small.

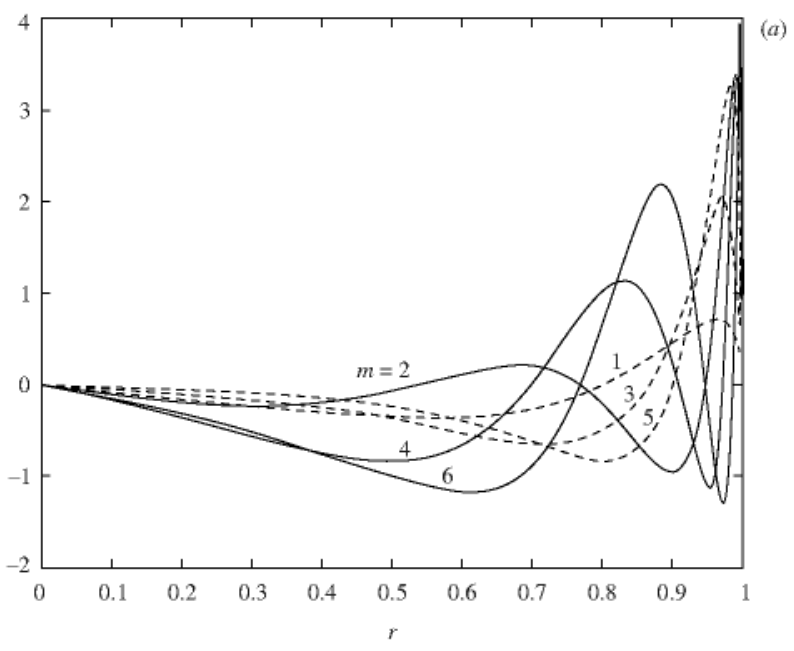




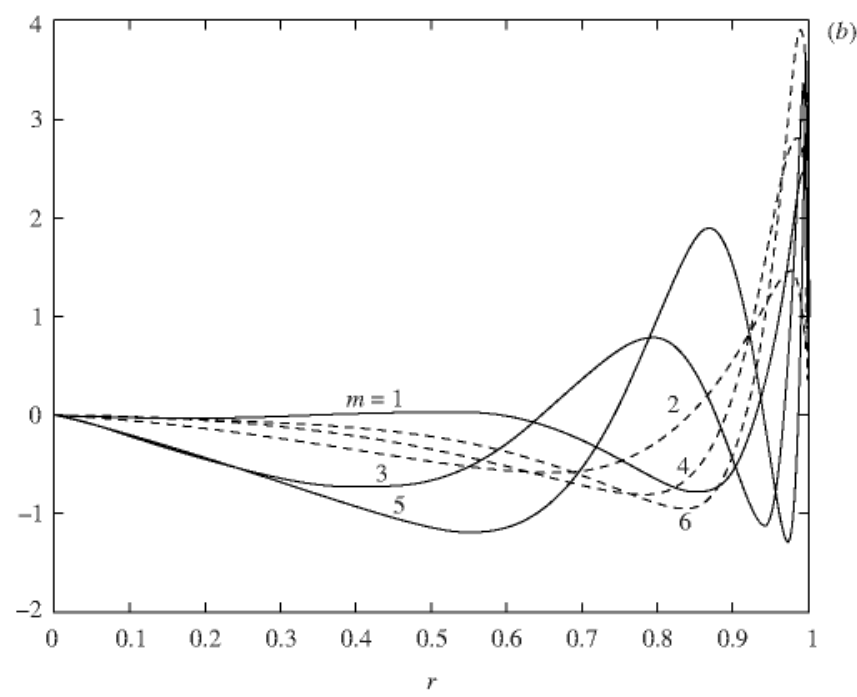

(b)

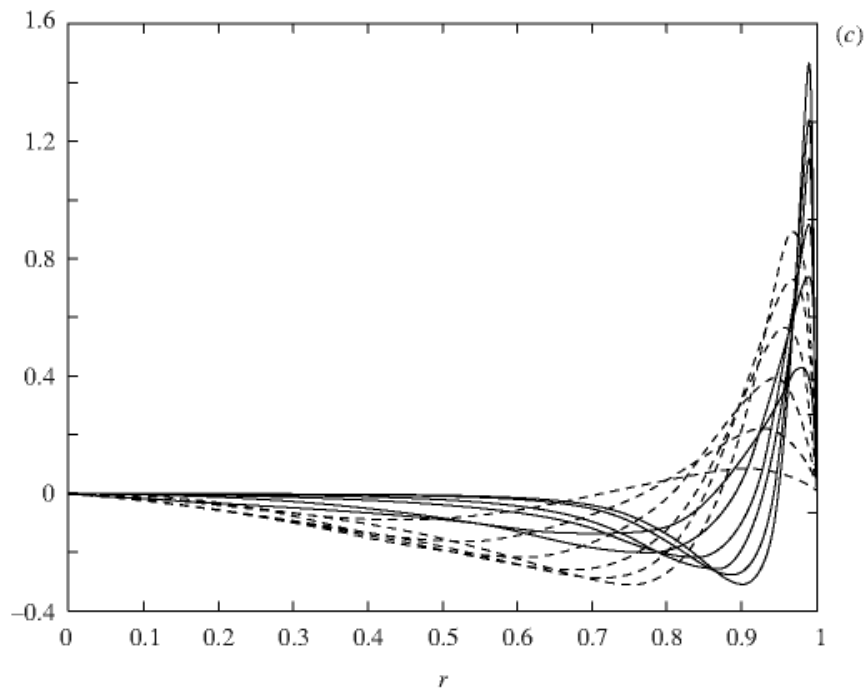

Figure 4 .

The forcing steady radial velocities near the disks, $G^{+}$and $G^{-}$, for $\Omega=\left(\Omega_{m}+\Omega_{m+1}\right) / 2$ and several values of $m$, where $\Omega_{m}$ is the $m$-th inviscid eigenfrequency, for $\Lambda=1$. (a) $a_{+}=a_{-}=1$, $\nu_{+}=\nu_{-}$and $m$ as indicated; $G^{+}=G^{-}$; in this case $(-) 10 G^{ \pm} / \Omega^{5 / 3},(----) G^{ \pm} / \Omega^{5 / 3}$. (b) As in (a) except $\nu_{+}=\nu_{-}-\pi$. (c) $a_{+}=1, a_{-}=0$, and $m=1, \ldots, 6$, such that the maximum of the curves increases and the minimum decreases as $m$ increases; $(\longrightarrow) G^{-} / \Omega^{5 / 3},(----)$ $G^{+} / \Omega^{5 / 3}$. 

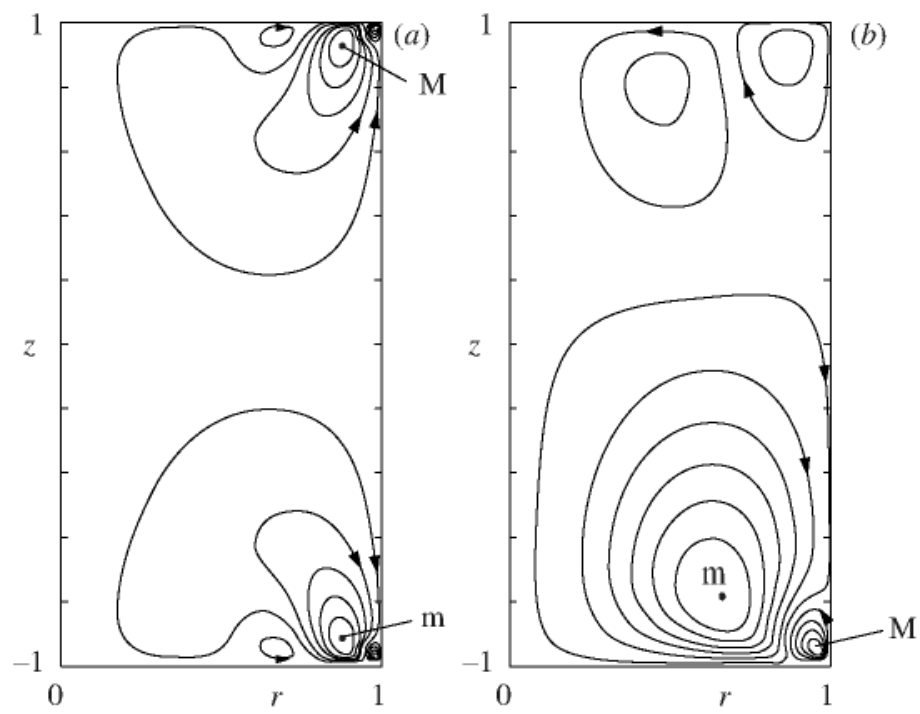

Figure 5.

Streamlines in the bulk for: (a) the forcing terms in Fig. 4a for $m=2(\Omega=10.1604$ ), and (b) the forcing terms in Fig. 4c for $m=1(\Omega=5.394)$. Represented are 10 equispaced streamlines, see Table 1 for extreme values.

d) If $a_{+} \cdot a_{-} \neq 0,\left|\nu_{+}-\nu_{-}\right|$is neither close to 0 not close to $\pi$ and $\Omega$ is large, then the associated streaming flow exhibits a unique toroidal eddy that is qualitatively appropriate to counterbalance thermocapillary convection if the Prandtl number is appropriately small, as we shall see in next sub-section.

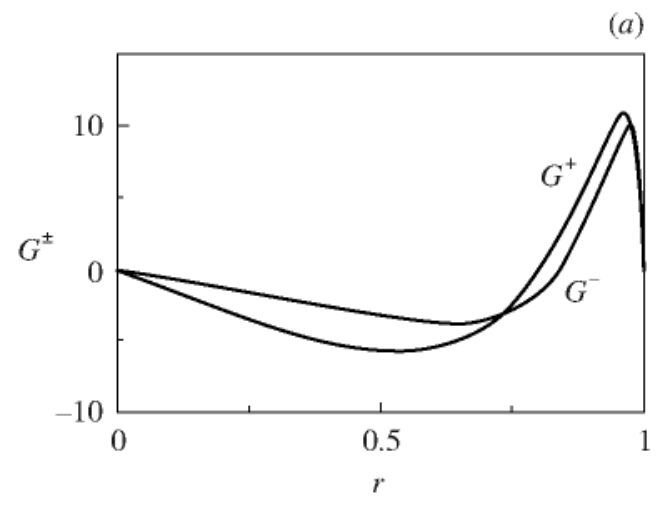


(b)

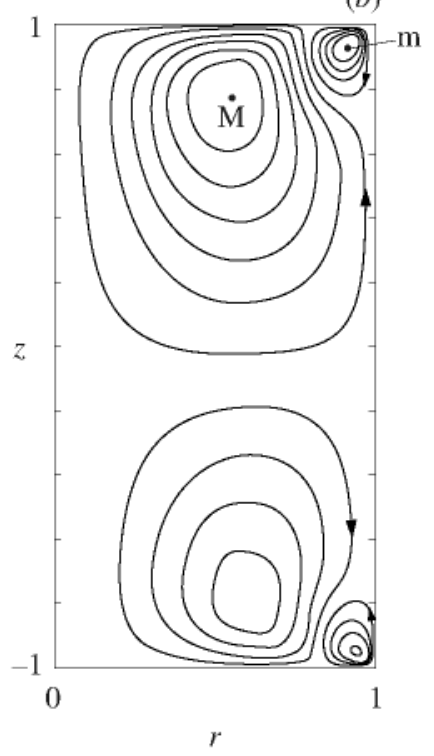

Figure 6 .

The forcing tangential velocities at the disks (a) and the streamlines (b) for $\Lambda=1, \Omega=4, a_{+}=1$ and $a_{-}=0$, represented are 10 equispaced streamlines, see Table 1 for extreme values.

\subsection{Counterbalancing thermocapillary convection of small Prandtl number}

We shall consider the asymptotic limit (2.78)-(2.79) that is realistic for liquid metals, whose Prandtl number $P$ is usually quite small (of the order of $10^{-2}$ ) and whose capillary Reynolds number $C^{-1}$ is usually quite large (of the order of $10^{4}$ ). In addition the thermal expansion coefficient $\lambda=\rho_{T}\left(T_{1}-T_{0}\right) / \rho_{0}$ usually satisfies

$$
\lambda \leq \sigma_{T}\left(T_{1}-T_{0}\right) / \sigma_{0}=\operatorname{Re} C^{2} .
$$

Then $\alpha=\lambda / C$ and $b=B / C$ are readily seen to satisfy (2.79) provided that $|B| \ll 1$ (as it is the case in microgravity conditions) and (2.78) holds. Then we only need to check the conditions in (2.78), that are readily seen to be satisfied if, e.g., $C \sim 10^{-4}, P \sim 10^{-2}, \Omega \sim 100$ and $R e \lesssim 10^{3}$. Now, as seen in $\S 2.4$, remark $\mathrm{g}$, in this limit we may take

$$
u^{a}=w^{a}=0, \quad \alpha=0, \quad G^{ \pm}=H^{ \pm}=0, \quad \phi=0
$$

in first approximation. As a consequence the streaming flow is driven only by the forcing tangential stress at the edge of the interface boundary layer, that is given by the second boundary condition in $(3.9), w_{r}^{s}=\psi-\operatorname{Re} \theta_{z}$. But, as we shall see below, the plot of $\psi$ in terms of the axial variable $z$ exhibits a fast oscillation (with a wavelength of the order of $\Omega^{-2 / 3}$ ) around a constant value $\psi_{0}$, and $\psi$ may be 


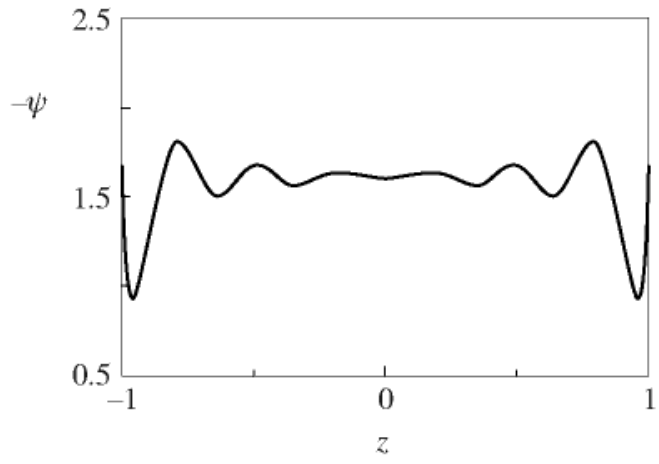

Figure 7.

The forcing steady tangential stress at the interface due to vibration $\psi$, for $\Lambda=1, \Omega=104$, $a=.001$ and $\nu_{+}-\nu_{-}=-\pi / 2$.

replaced by $\psi_{0}$ in first approximation (for the effect of two neighboring oscillations will approximately cancel each other). If this is done, and the constant $\psi_{0}$ (that may be controlled through the vibration parameters) is chosen to be $\psi_{0}=-R e / \Lambda$, then the problem (3.3)-(3.9) has the exact solution $u^{s} \equiv w^{s} \equiv 0, \theta=-1 / \Lambda$ (recall that we are imposing (3.21)). This explains the fairly good counterbalancing results that will be obtained below.

For illustration, the following representative values of $P, R e, \Lambda$ and $\Omega$ will be considered

$$
P=.02, .05, .1 \text { and } .2, \quad R e=100 \text { and } 1000, \quad \Lambda=1, \quad \Omega=104 .
$$

In addition the phase shift $\nu_{+}-\nu_{-}$will be always taken equal to $\pm \pi / 2$ for $|\psi|$ to be as large as possible (see (3.16)); the sign of $\nu_{+}-\nu_{-}$will be selected in each case for $\psi$ to have the appropriate sign (see (3.16)). Finally, the forcing amplitudes, that will be selected to be equal, act as the controlling parameter; in fact, the parameter

$$
k=10^{6} a^{2} / R e, \quad \text { with } a=a_{+}=a_{-},
$$

will be considered, instead of $a$, as the controlling parameter.

In Fig. 7 we give the forcing tangential stress due to vibration $\psi$ for $\Lambda=1$, $\Omega=104, a=.001$ and $\nu_{-}-\nu_{+}=\pi / 2$. If $\Omega$ is maintained essentially constant (and midway in between of two neighboring natural frequencies) and $\Lambda$ is varied then the wavelength and amplitude of the oscillation in the plot of $\psi$ decrease as $\Lambda$ increases.

The graphs in Fig. 8 show the axial velocity and the temperature at the free surface, $w_{\text {surf }}$ and $\theta_{\text {surf }}$, for $\Lambda=1, \Omega=104, \nu_{-}-\nu_{+}=\pi / 2, R e=1000$ and $P=$ $.02, .05, .1$ and .2 . Plots (a) and (b) correspond to $k=0$ (pure thermocapillary flow without vibration), and plots (c) and (d) to $k=.61$, that is an optimum value of the vibration amplitude, which drastically reduces the strength of thermocapillary flow. This effect is slightly stronger for low $P$ : if $P=.02$, then $\left|w_{\text {surf }}\right| \max =174$ 


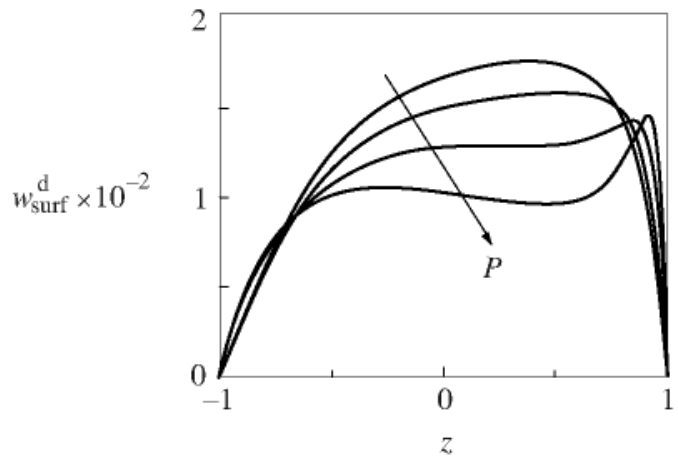

(a)
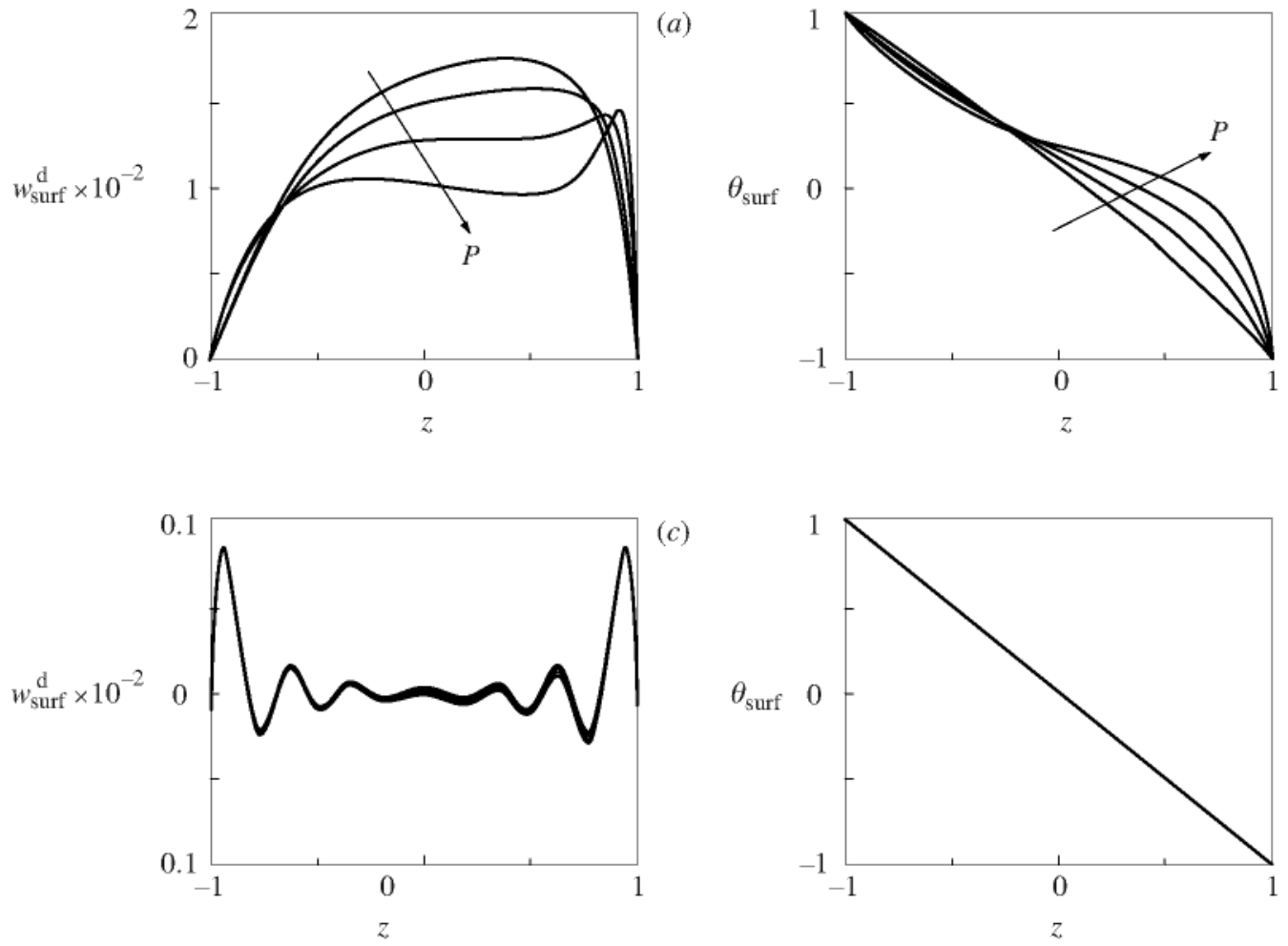

(d)

Figure 8.

Axial velocity and temperature at the free surface vs. the axial coordinate for $R e=1000, \Lambda=1$, $\Omega=104$ and $P=.02, .05, .1$ and $.2 .(\mathrm{a}, \mathrm{b}) k=0$ and $(\mathrm{c}, \mathrm{d}) k=.61$.

for $k=0$, and $\left|w_{\text {surf }}\right| \max =8.19$ for $k=.61$ (a $95 \%$ reduction); if $P=.2$, then $\left|w_{\text {surf }}\right| \max =143$ for $k=0$, and $\left|w_{\text {surf }}\right| \max =8.08$ for $k=0.61$ (a $94 \%$ reduction). As shown in plot (d), for $k=.61$ the temperature field is reduced almost completely to the conduction state (this effect is due to the extreme convection reduction).

Figure 9 corresponds to the same values of the parameters as in Fig. 8 (including the optimum value $k=.61$ which remains unchanged) except for the effective Reynolds number that now is $R e=100$. The effect in weakening the thermocapillary flow is now even stronger. For $R e=100$ convection is weaker than for $R e=1000$, thus the effect of $P$ is even smaller. The variation of $R e$ does not produce qualitative differences on the results. Therefore, only the value $R e=1000$ is considered below.

If $\Omega$ is kept essentially constant (and midway in between of two neighboring natural frequencies), and $R e$ and $P$ are kept fixed, then counterbalancing results improve as $\Lambda$ increases (and worsen as $\Lambda$ decreases). For example, if $R e=10^{3}$, $P=.02$ and $\left(\Lambda, \Omega, \nu_{+}-\nu_{-}\right)=(.5,106, \pi / 2)$ then for $k=1.17$ (that is now an 


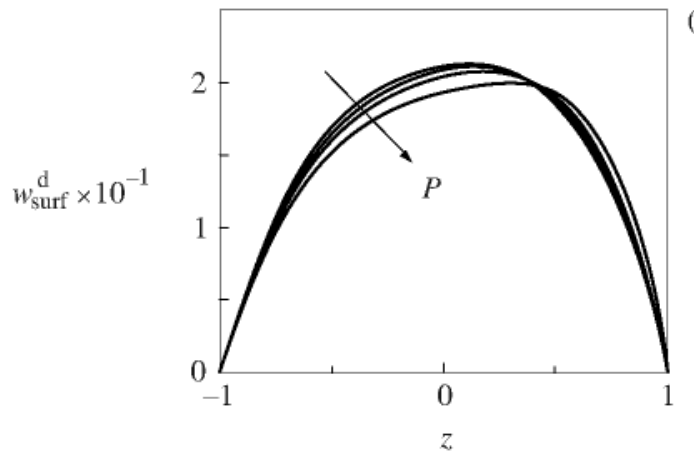

(a)

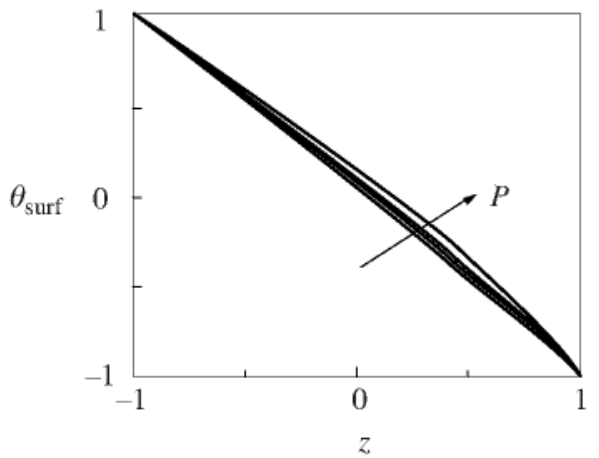

(b)
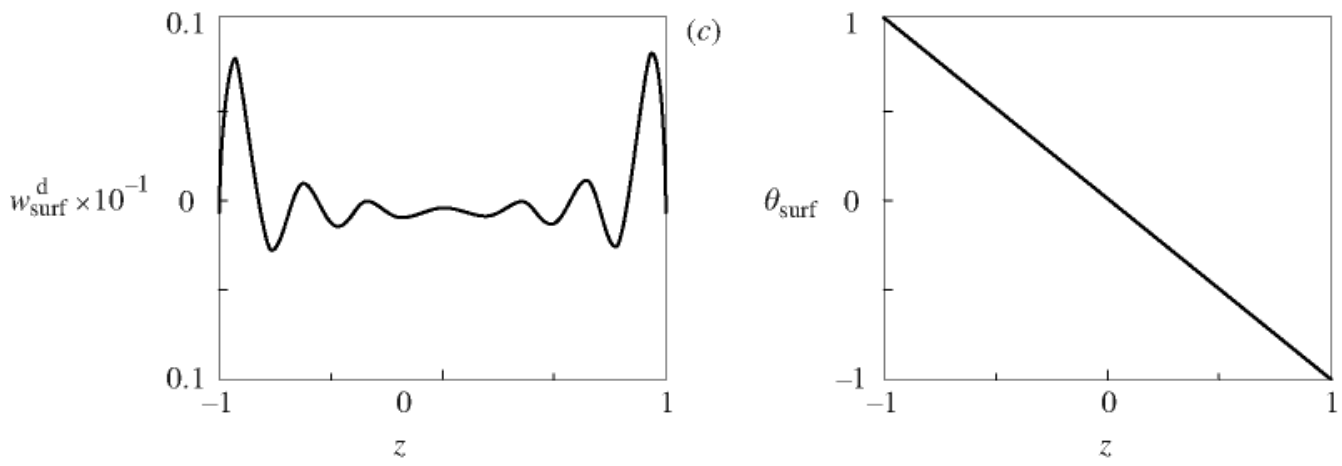

(d)

Figure 9.

Axial velocity and temperature at the free surface vs. the axial coordinate for $R e=100, \Lambda=1$, $\Omega=104$ and $P=.02, .05, .1$ and $.2 .(\mathrm{a}, \mathrm{b}) k=0$ and $(\mathrm{c}, \mathrm{d}) k=.61$.

optimum value for counterbalancing) we obtain a $91 \%$ reduction of $\left|w_{\text {surf }}\right|$ max ; if $\left(\Lambda, \Omega, \nu_{+}-\nu_{-}\right)=(2,104,-\pi / 2)$ then for $k=.312$ we obtain a $96 \%$ reduction of $\left|w_{\text {surf }}\right| \max$. Notice that this is in accordance with the dependence on $\Lambda$ of the forcing function $\psi$ that was described above.

When the value of $k$ is increased over the optimum value a flow pattern opposite to the pure thermocapillary flow is obtained. In Figs. 10 and 11 we give results for $\Lambda=1, \Omega=104, \nu_{-}-\nu_{+}=\pi / 2, P=.02, R e=1000$ and three values of $k$ : $k=0$ (pure thermocapillary flow), $k=.61$ (optimum value to counterbalance the thermocapillary flow) and $k=1.2$ (opposite flow field). The plots in Fig. 10 show

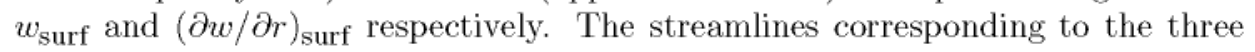
cases are shown in Fig. 11; the maximum of the stream function modulus in plots (a) $(k=0)$ and (c) $(k=1.2)$ are 19.2 and 20.8 respectively, whereas in plot (b) $(k=.61)$ the maximum is .133 , showing that the flow is much weaker in this case.

Notice that Fig. 11c looks like the mirror image of Fig. 11a. This was to be expected because in both cases (i) $\psi$ may be replaced by the constant $\psi_{0}=$ 

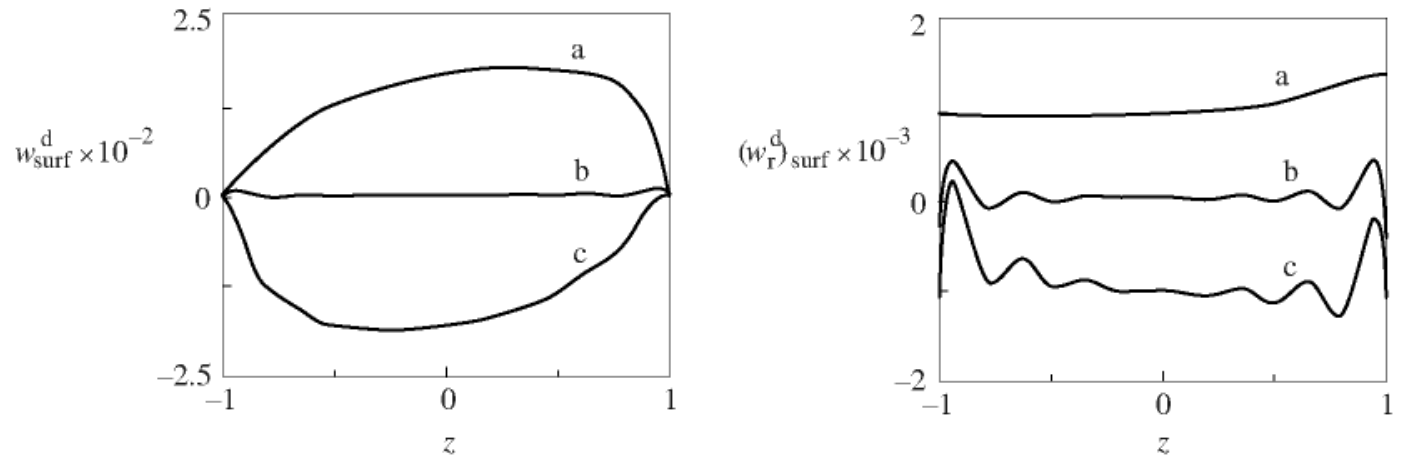

Figure 10 .

Axial velocity and its radial derivative at the free surface vs. the axial coordinate for $\Lambda=1$, $\Omega=104, R e=1000, P=.02$. (a) $k=0$, (b) $k=.61$ and (c) $k=1.2$.
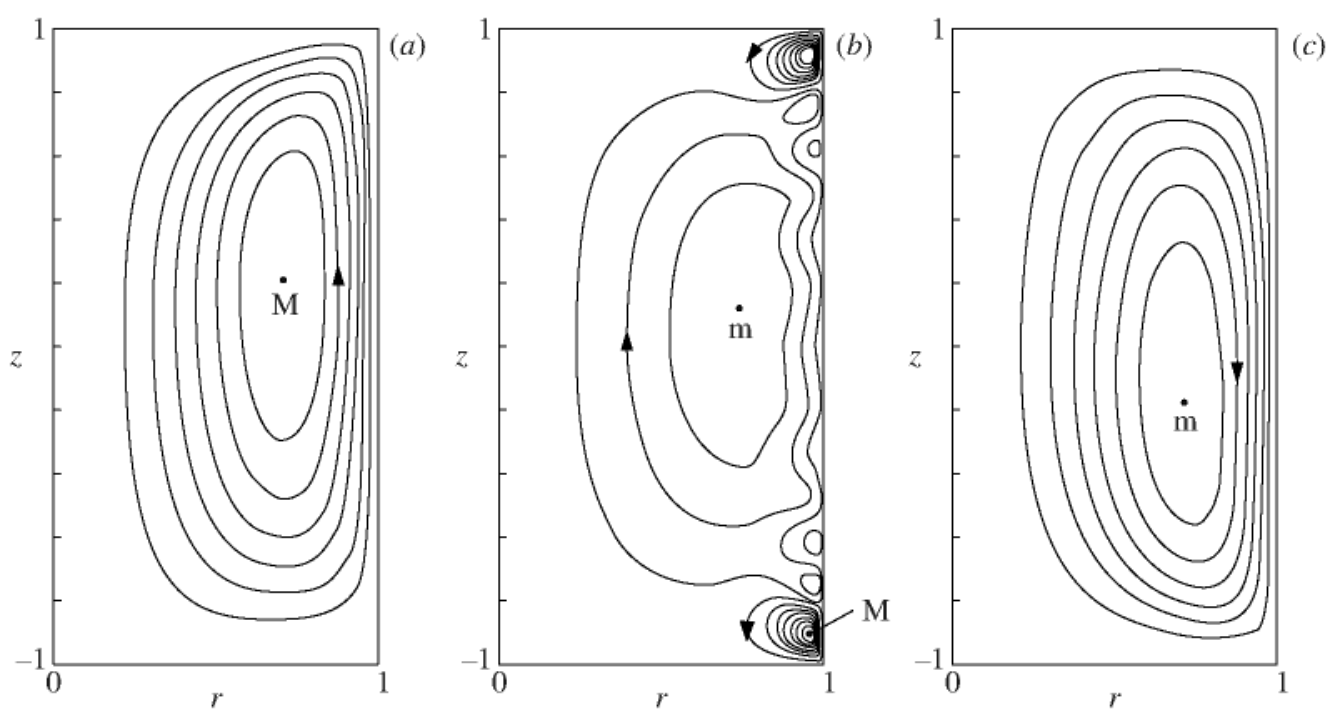

Figure 11.

Streamlines in the bulk for $\Lambda=1, \Omega=104, R e=1000$ and $P=.02$. (a) $k=0$, (b) $k=.61$ and (c) $k=1.2$. Represented are 6 equispaced streamlines in (a) and (c), and 10 in (b), see Table 1 for extreme values.

$-1.65 k$ Re (see Fig. 7 and take into account (3.16) and (3.22) and our remark at the beginning of this sub-section) and (ii) $\theta_{z} \simeq-1$ (see Fig. 8d). Then, in the two cases considered in Figs. 11a and 11c, the boundary conditions (3.9) are, in 


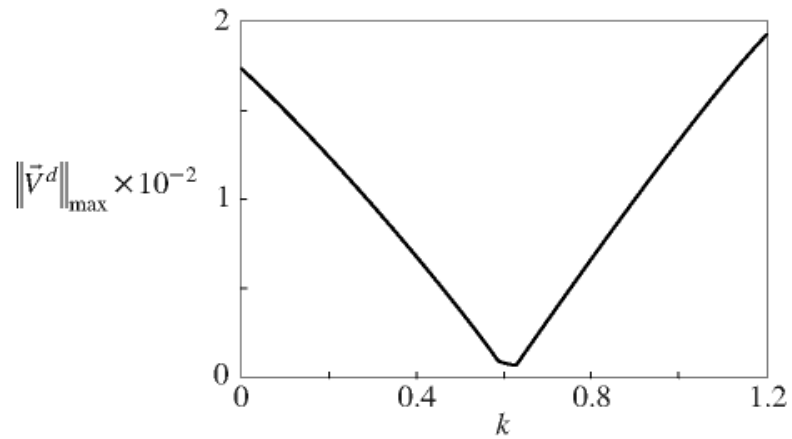

Figure 12.

Maximum velocity modulus in the liquid bridge in terms of the controlling parameter $k$ for $\Lambda=1$, $\Omega=104, R e=1000$ and $P=.02$.

Table 1. Streamfunction maximum and minimum and their locations in the figures representing streamlines. $\Psi$ is defined by $u=\frac{-1}{r} \frac{\partial \Psi}{\partial z}, w=\frac{1}{r} \frac{\partial \Psi}{\partial r}$

\begin{tabular}{||l||c|c|c||c|c|c||}
\hline \hline & $\Psi_{\max }$ & $r$ & $z$ & $\Psi_{\min }$ & $r$ & $z$ \\
\hline \hline Fig. 3a & 1.07 & 0.551 & 0.737 & -1.07 & 0.551 & -0.737 \\
Fig. 3b & 12.8 & 0.859 & -0.709 & -12.8 & 0.859 & 0.709 \\
Fig. 5a & 0.0824 & 0.874 & 0.932 & -0.0824 & 0.874 & -0.932 \\
Fig. 5b & 0.0852 & 0.950 & -0.957 & -0.107 & 0.649 & -0.794 \\
Fig. 6b & 0.239 & 0.551 & 0.784 & -0.182 & 0.929 & 0.942 \\
Fig. 11a & 19.2 & 0.716 & 0.206 & 0. & boundary & boundary \\
Fig. 11b & 0.133 & 0.954 & -0.915 & -0.0638 & 0.730 & 0.123 \\
Fig. 11c & 0. & boundary & boundary & -20.8 & 0.716 & -0.177 \\
\hline \hline
\end{tabular}

first approximation,

$$
u=0, \quad w_{r} \simeq 1000 \text { and }-980 \text { respectively, at } r=1 .
$$

Consequently, each flow may be obtained from the other one by a symmetry on the plane $z=0$ in first approximation.

Finally, in Fig. 12 we give the maximum of the velocity modulus in the liquid bridge as a function of $k$, for $\Lambda=1, \Omega=104, \nu_{-}-\nu_{+}=\pi / 2, P=.02$ and 
$R e=1000$. This figure illustrates how the flow pattern can be controlled through the parameter $k$, that is, through the vibration amplitude. Notice that the selected optimum value of $k$ (that was defined as $10^{6} a^{2} / R e$ ) in this case is .61 ; then $a=$ $\left|\beta_{+}\right|=\left|\beta_{-}\right|=2.47 \cdot 10^{-2}$ and the vibrating amplitude is $\sqrt{C}\left|\beta_{ \pm}\right|=7.81 \cdot 10^{-4}$ if $C=10^{-3}$. In the remaining cases considered above, the vibrating amplitudes required to counterbalance thermocapillary convection are also quite small, of the order of $10^{-3} \sqrt{\operatorname{ReC}}$.

\section{Concluding remarks}

The interaction of thermocapillary stresses, steady forcing due to non-resonant, axisymmetric, mechanical vibrations and thermal expansion effects have been considered for low-viscosity liquid bridges (i.e., with large capillary Reynolds number, $C^{-1}$ ) and low nondimensional (based on the capillary characteristic time $\left(\rho R^{3} / \sigma_{0}\right)^{1 / 2}$ ) vibrating frequencies (i.e., such that $\Omega \ll C^{-1}$ ). We have considered a model-half-zone liquid bridge (temperatures of the disks are held fixed and the free surface is thermally insulated). We have obtained an asymptotic model, posed by (3.3)-(3.9) that accounts for the interaction of these effects in first approximation. This model has been numerically integrated in $\S 3$ to obtain steady streaming flows in the bulk in several limiting cases. In isothermal conditions we have considered several cases in which the vibrating field is standing in first approximation and obtained several flow patterns that have been compared with existing experimental results in the literature. In non-isothermal conditions we have only considered the asymptotic limit (2.78)-(2.79), that have been seen to be realistic for liquid metals. In this limit, we have shown that the effect of thermocapillary stress can be counterbalanced almost completely by appropriate choices of the vibrating amplitude (that appeared to be quite small) and frequencies, and of the difference of the vibrating phases of the disks; in fact, the steady forcing terms due to vibration depend crucially on the latter (and this could be somewhat surprising at first sight). Counterbalancing results improve as either the Prandtl number decreases or the slenderress and/or the forcing frequency increase.

In addition to providing a qualitative and quantitative explanation of the counterbalancing effect, we expect our results to serve as a guide to experimentalists. For example, the authors of [13] reported that no counterbalancing effect was obtained for low-viscosity liquids at low frequencies; our results show that if both disks were vibrated (they vibrated only one disk) with appropriate vibrating parameters (and special care on vibrating phases), then the required counterbalancing effect could have been obtained at not-so-large frequencies.

Finally, let us mention the limitations of the results above:

a) The approximate model (3.3)-(3.9) and the approximations (3.21) result from neglecting other terms that are smaller than those considered when the tangential stress at the interface (in the boundary condition (3.9)) is such that $\left|\psi-\operatorname{Re} \theta_{z}\right| \sim$ $R e$. But in $\$ 3.4$ counterbalancing is so effective precisely because $\left|\psi-R e \theta_{z}\right|$ is 
fairly small as compared to Re. Therefore the neglected terms could affect the detailed quantitative results in Figs. $8 \mathrm{c}, 9 \mathrm{c}$ and $11 \mathrm{~b}$; of course the neglected terms do not affect the main underlying result, namely, that convection is drastically reduced by vibration.

b) We have considered a first distinguished limit, $\Omega \sim 1$, that applies whenever $\Omega \ll C^{-1}$. A second distinguished limit, $\Omega \sim C^{-1}$, that applies whenever $1 \ll$ $\Omega \ll C^{-3}$, will be considered elsewhere [22]. This second limit is of great interest. because: (i) as explained in $\$ 3$, best counterbalancing effects are obtained when the vibrating frequency is as large as possible, and (ii) more general thermal boundary conditions may be considered, as explained in (c).

c) In connection with (b), as explained in $\$ 3$, the most effective steady forcing term due to vibration is associated with the function $\psi$, giving $\left(\partial w^{s} / \partial r\right)$ at the free boundary. Since the function $\psi$ is symmetric on the axial variable (see (3.16)), vibrations may counterbalance only axially symmetric thermocapillary stresses in the limit considered in this paper . Again, in the second distinguished limit, $\Omega \sim C^{-1}$, vibrations may produce also steady forcing terms that are appropriate to annihilate axially symmetric thermocapillary flows (that are the most interesting ones in applications concerning crystal growth processes) resulting from axially antisymmetric thermocapillary stresses.

d) The results in $\S 3$ are concerned only with axisymmetric steady flows, that might be unstable. Our numerical calculations allow us to predict that no bifurcations to other branches of steady axisymmetric steady flows take place, at least in the parameter range we have considered; but bifurcations to non-axisymmetric steady flows or to time dependent ones cannot be excluded.

\section{References}

[1] J. C. Melrose, Model calculations for capillary condensation, A. I.Ch. E. I 12 (1966), 986.

[2] J.N. Zasadzinski, J.B. Sweeney, H.T. Davis and L.E. Scriven, Finite element calculation of fluid menisci and thin-films in model porous media, $J$. Colloid Interface $S_{c}$. 119 (1987), 108.

[3] T.Y. Clen and J. Tsamopoulos and R.J. Good, Capillary bridges between parallel and non-parallel surfaces and their stability, J. Colloid Interface Sci. 151 (1992), 49.

[4] J. Tsamopoulos, T.-T. Chen and A. Borkar, Viscous oscillations of capillary bridges, $J$. Fluid Mech. 235 (1992), 579-609.

[5] F. Preiser, D. Schwabe and A. Sharman, Steady and oscillatory thermocapillary convection in liquid columns with tree cylindrical surface, J. Fluid Mech. 126 (1983), 545-567.

[6] Y. Kamotani, S. Ostrach and M. Vargas, Oscillatory thermocapillary convection in a simulated floating-zone configuration, $J$. Crystal Growth 66 (1984), 83-90.

[7] M. Jurish and W. Löser, Analysis of periodic non-rotational W striations in $M_{0}$ single crystals due to nonsteady thermocapillary convection, J. Cryst. Growth 102 (1900), 214222 .

[8] W.W. Fowlis and G.O. Roberts, Confinement of thermocapillary floating zone by uniform rotation, J. Cryst. Growth 74 (1986) 301-320.

[9] G.D. Robertson Jr. and D. O'Connor, Magnetic field effects on float-zone Si crystal growth. III. Strong axial fields, $J$. Cryst. Growth 88 (1988) 148-158. 
[10] A. Cröll, P. Dold and K.W. Benz, Segregation of si floating-zone crystals grown under microgravity and in a maguetic field, $J$. Cryst. Growth 137 (1994), 95-101.

[11] R.F. Dressler and N.S. Sivakumaran, Non-contaminating method to reduce Marangoni convection in microgravity float zones, J. Cryst. Growth 88 (1988) 148-158.

[12] C.W. Lan and S. Kou, Floating-zone crystal growth with a heated and immersed shaperexperiments, J. Cryst. Grouth 108 (1991) 541-548.

[13] A.V. Anilkumar, R.N. Grugel, X.F. Shen, C.P. Lee and T.G. Wang, Control of thermocapillary convection in a liquid bridge by vibration, $J$. Appl. Phys. 73 (1993), 4165-4170.

[14] G.G. Stokes, On the theory of oscillatory waves, Trans. Camb. Phit. Soc. 8 (1847), 441-455.

[15] A.D.D. Craik, The drift velocity of water waves, J. Fluid Mech. 116 (1982), 187-205.

[16] Z. Zapyanov, Zh. Kozhoukharova and A. Iordanova, On the hydrodynamic interaction of two circular cylinders oscillating in a viscous fluid, Z. Angew. Math. Phys 39 (1988), $204-220$.

[17] N. Riley, Acoustic streaming about a cylinder in orthogonal beams, J. Fluid Mech. 242 (1992), 387-394.

[18] B. Yan, D.B. Ingham and B.R. Morton, Streaming flow induced by an oscillating cascade of circular cylinders, J. Flutid Mech. 252 (1993), 147-171.

[19] S. Biringen and L.J. Peltier, Computational study of 3-D Bénard convection with gravitational modulation, Phys. Fluids A 2 (1990), 279-283.

[20] A. Faroog and G.M. Homsy, Streaming flows due to $g$-jitter-induced natural convection, J. Flatid Mech. 271 (1994) 351-378.

[21] A.D. Mysthkis, V.G. Babskii, N.D. Kopachevskii, L.A. Slobozhauin and A.D. Tyuptsov, Low-gravity Fluid Mechanics, Springer-Verlag, Berlin 1987.

[22] J.A. Nicolás, D. Rivas and J.M. Vega, On the steady streaming flow due to high-frequency vibration in nearly-inviscid liquid bridges, Submitted to J. Fituid Mech., 1997.

[23] H. Schlichting, Berechuung ebener periodischer Grenzschichtströmungen, Phys. Z 33 (1932), $327-335$.

[24] M.S. Longuet-Higgins, Mass transport in water waves, Phil. Trons. Roy. Soc. A 245 (1953), 535-581.

[25] J.A. Nicolás and J.M. Vega, Weakly nonlinear oscillations of axisymmetric liquid bridges, J. Fluid Mech. 328 (1996), 95-128.

[26] M. Higuera, J.A. Nicolás and J.M. Vega, Linear oscillations of weakly dissipative axisymmetric liquid bridges, Phys. Fluids A 6 (1994), 438-450.

[27] G.K. Batchelor, An Introduction to Flaid Dynamics, Cambridge Univ. Press 1967.

[28] S.V. Patankar, Numerical Heat Transfer and Fluid Flow, Hemisphere, Washington D.C. 1980 .

[29] S.D. Connell and P. Stow, The pressure correction method, Computers \& Fluids 14 (1986), $1-10$.

[30] J.P. Van Doormaal and G.D. Raithby, Enhancements of the SIMPLE method for predicting incompressible fluid flows, Numerical Heat Transfer 7 (1984), 147-163.

[31] W.J. Minkowycz, E.M. Sparrow, G.E. Schneider and R.H. Pletcher, Handbook of Numerical Heat Transfer, John Wiley \& Sons 1988.

[32] F.M. Harlow and J.E. Welch, Numerical calculation of time-dependent incompressible flow of fluid with a free surface, Phys. Fluids 8 (1965), 2182-2189.

[33] D.J. Mollot, J. Tsamopoulos, T.Y. Clien and N. Asligriz, Nonlinear dynamics of capillary bridges: experiments, J. Fluid Mech. 255 (1993), 411-435. 
José A. Nicolás

E.T.S.I. Aeronáuticos

Universidad Politécnica de Madrid

28040-Madrid. SPAIN

Damián Rjvas

E.T.S.I. Aeronáuticos

Universidad Politécnica de Madrid

28040-Madrid. SPAIN
Jusé M. Vega

E.T.S.I. Aeronáuticos

Universidad Politécnica de Madrid

28040-Madrid. SPAIN 